\title{
Energy-Efficient Zero-Forcing Precoding Design for Small-Cell Networks
}

\author{
Quang-Doanh Vu, Le-Nam Tran, Member, IEEE, Ronan Farrell, Member, IEEE, \\ and Een-Kee Hong, Senior Member, IEEE
}

\begin{abstract}
We consider small-cell networks with multipleantenna transceivers and base stations (BSs) cooperating to jointly design linear precoders to maximize the network energy efficiency, subject to a sum power and per-antenna power constraints at individual BSs, as well as user-specific quality of service $(\mathrm{Q} O S)$ requirements. Assuming zero-forcing precoding, we formulate the problem of interest as a concave-convex fractional program to which we proposed a centralized optimal solution based on the prevailing Dinkelbach algorithm. To facilitate distributed implementations, we transform the design problem into an equivalent convex program using Charnes-Cooper's transformation. Then, based on the framework of alternative direction method of multipliers (ADMM), we develop a decentralized algorithm, which is numerically shown to achieve fast convergence. Since BSs are generally power-hungry, it may be more energy-efficient if some BSs can be shut down, while still satisfying the QoS constraints. Toward this end, we investigate the problem of joint precoder design and BS selection, which is a mixed Boolean nonlinear program, and then provide an optimal solution by customizing the branch-andbound method. For real-time applications, we propose a greedy algorithm which achieves near-optimal performance in polynomial time. Numerical results are provided to demonstrate the effectiveness of the proposed algorithms.
\end{abstract}

Index Terms-Small-cell networks, energy efficiency, MIMO, joint design, ADMM, branch-and-bound.

\section{INTRODUCTION}

$\mathbf{T}$ HE RECENT explosive growth of user terminals, e.g. smart phones and wireless modems has led to the so-called data capacity crunch on mobile networks. Small-cell networks, where low-cost low-power base stations (BS) are deployed, have been considered as a promising technique to solve this problem due to their capability of boosting coverage

Manuscript received May 1, 2015; revised August 18, 2015 and October 7, 2015; accepted November 12, 2015. Date of publication November 23, 2015; date of current version February 12, 2016. This work was supported in part by the International Research \& Development Program of the National Research Foundation of Korea (NRF) funded by the Ministry of Science, ICT \& Future Planning (Grant number: NRF-2012K1A3A1A26034927); and in part by a research grant from Science Foundation Ireland and is co-funded by the European Regional Development Fund under Grant 13/RC/2077. The associate editor coordinating the review of this paper and approving it for publication was S. Durrani.

Q.-D. $\mathrm{Vu}$ was with the School of Electronics and Information, Kyung Hee University, Seoul, South Korea. He is now with the Department of Communications Engineering and Centre for Wireless Communications, University of Oulu, Oulu 90014, Finland (e-mail: quvu@ee.oulu.fi).

L.-N. Tran and R. Farrell are with the Department of Electronic Engineering, Maynooth University, Maynooth, Co. Kildare, Ireland (e-mail: ltran@eeng.nuim.ie; rfarrell@eeng.nuim.ie).

E.-K. Hong is with the School of Electronics and Information, Kyung Hee University, Seoul, South Korea (e-mail: ekhong@khu.ac.kr).

Color versions of one or more of the figures in this paper are available online at http://ieeexplore.ieee.org.

Digital Object Identifier 10.1109/TCOMM.2015.2502941 and capacity [1]-[3]. In typical usage scenarios, user terminals lie in an overlapping coverage of multiple BSs (macro BS and small-cell BSs). Interesting problems arising from this context may include how the BSs collaborate to optimize a performance measure, e.g. maximizing sum rate or minimizing total transmit power.

It is well known that multi-input multi-output (MIMO) transmission is a powerful technique to improve capacity of wireless communication systems without requiring extra bandwidth or transmit power. In fact, MIMO technology is currently adopted in all current broadband wireless communication standards, e.g. LTE, WLAN. In small-cell networks where BSs are equipped with multiple antennas, BSs can cooperate to serve all the users by jointly designing their transmit precoders to achieve a specific design criterion. For example, a linear precoder design which maximizes utility function was investigated in [4]. The authors in [5], [6] considered a joint beamformer design problem to minimize total power consumption. The recent work of [7] studied full-duplex transmission in small-cell networks and jointly designed beamfomers to maximize spectral efficiency.

Energy efficiency has recently become an emerging important metric due to a growing attention to greenhouse gas emissions contributed by cellular networks. In fact, improving energy efficiency has been the main focus in a large number of recent works under different scenarios such as OFDM [8], MIMO transmission [9], massive MIMO [10], sensor networks [11] and CoMP [6], [12]-[14]. Note that energy efficiency is defined as the ratio of the aggregate throughput to the total power consumption in a network, which basically means the problem of energy efficiency maximization (EEmax) belongs to a class of fractional programs. In most of the above mentioned works related to energy efficiency, the authors formulated their considered problem as a concave-convex fractional program, and then obtained global optimality by Dinkelback's algorithm, e.g. [14], [15], or bisection method, e.g. [8], [9], [12], which requires solving a sequence of parameterized convex problems [15]. This approach results in a kind of outer-inner loop algorithm which is easily handled by a central node, however, it is not convenient for distributed implementation.

In this paper we first consider small-cell networks with a fixed configuration where all the BSs are assumed to be active. Note that in the multiuser MIMO (MU-MIMO) systems, the optimal downlink transmission strategy is dirty paper coding, which is a nonlinear precoding technique that requires high complexity to implement [16]. To relax the complexity issue we therefore assume linear precoding for the BSs, and consider the joint design of linear precoders to maximize the network energy 
efficiency, subject to user-specific QoS requirements and both sum power and per-antenna power constraints (PAPC) at BSs. Unfortunately, finding optimal linear precoders in the considered problem is very challenging due to the existence of multiuser interference. Consequently, suboptimal linear precoding schemes such as those based on zero-forcing (ZF) [17] and minimum mean square error (MMSE) [18] criteria have been widely used. In this paper we adopt zero-forcing precoding which enables us to formulate the EEmax problem as a concave-convex fractional program. In fact, $\mathrm{ZF}$ technique was also used in [6], [14] for designing energy-efficient coordinated transmit precoding. However, for simplification, the work in [6] fixed the user data rates, and thus the EEmax problem reduces to a power minimization one. Similarly, the authors in [14] assumed a fixed-phase $\mathrm{ZF}$ beamforming to convert the precoder design problem into the one of power allocation. Thus, the energy efficiency performances yielded by these approaches are far from those obtained by our proposed designs as shown in Section V. For multicell settings with macro BSs, the work of [12], [13] designed energy efficient precoding for multiuser MIMO based on MMSE technique. We remark that the methods aforementioned are basically centralized.

For the formulated EEmax problem we propose an algorithm based on Dinkelbach's method in which convex subproblems are iteratively solved. In particular we present two methods to solve the subproblems. In the first method we use dual decomposition technique and express the optimal precoders in a semiclosed form, which provides useful insights into the EEmax problem. However, dual decomposition is generally known to exhibit slow convergence since it is based on subgradient methods. To address this problem we equivalently transform the subproblems into a more standard convex program which can be solved much efficiently by modern interior-point solvers. Next, we convert the EEmax problem to a single equivalent convex program using Charnes-Cooper's transformation [19], and then develop a distributed algorithm via the alternating direction method of multipliers (ADMM) [20].

In the EEmax problem, beside data dependent power, data independent power, e.g. the power required to activate RF circuits in BSs, also contributes to the transmission cost. This means appropriately blocking the transmission from a BS to a user and/or switching off a BS may improve the network energy efficiency. It is worth noting that, in a small-cell network, a user can receive data from macro BS and small-cell BSs. Thus, turning off some small-cell BSs does not interrupt the data transmission. This motivates us to investigate the problem of joint precoder design and BS selection (JPBS). To this end we formulate the JPBS problem as a mixed Boolean fractional program, and customize the branch-and-bound $(\mathrm{BnB})$ method [21], [22] which possibly achieves an optimal solution with much reduced computational complexity compared to a bruteforce search. For practical implementation we propose a lowcomplexity suboptimal algorithm based on the idea of greedy selection which is numerically shown to yield near-optimal performance in polynomial time.

The rest of the paper is organized as follows. System model and the formulation of the EEmax problem are described in Section II. In Section III we present the proposed centralized

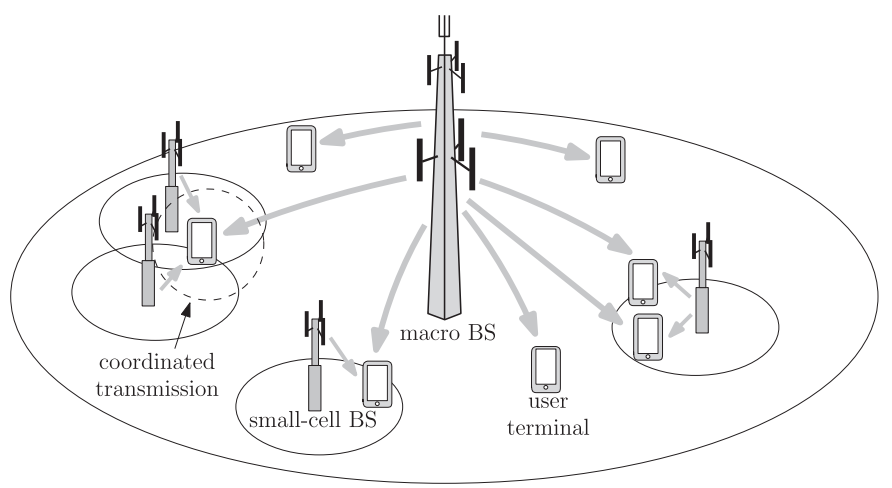

Fig. 1. An example of small-cell deployment cellular network.

and distributed algorithms. Section IV introduces the JPBS problem and describes the proposed optimal algorithm and the suboptimal low-complexity design. Numerical results are provided in Section V, and Section VI concludes the paper.

Notation: Standard notations are used in this paper. Bold lower and upper case letters represent vectors and matrices, respectively; $\mathbf{X}^{H}$ and $\mathbf{X}^{T}$ are Hermitian and normal transpose of $\mathbf{X}$, respectively; $\operatorname{Tr}(\mathbf{X})$ and $|\mathbf{X}|$ are the trace and determinant of $\mathbf{X}$, respectively. $\mathbf{I}_{M}$ and $\mathbf{0}_{M \times N}$ represent an $M \times M$ identity matrix and $M \times N$ zero matrix, respectively; $[\mathbf{x}]_{i}$ is the $i$ th entry of vector $\mathbf{x} ;[\mathbf{X}]_{i, j}$ is the entry at the $i$ th row and $j$ th column of $\mathbf{X}$; $\operatorname{diag}(\mathbf{x})$, where $\mathbf{x}$ is a vector, denotes a diagonal matrix with elements $\mathbf{x}$; $\operatorname{diag}(\mathbf{X})$, where $\mathbf{X}$ is a square matrix, denotes a vector of its diagonal elements. The notation $\mathbf{X} \succeq 0$ means that $\mathbf{X}$ is positive semidefinite. For ease of description, we also use "MATLAB notation" throughout the paper. Specifically, when $\mathbf{H}_{1}, \ldots, \mathbf{H}_{k}$ are matrices with the same number of rows, $\left[\mathbf{H}_{1}, \ldots, \mathbf{H}_{k}\right]$ denotes the matrix with the same number of rows obtained by staking horizontally $\mathbf{H}_{1}, \ldots$, and $\mathbf{H}_{k}$. When $\mathbf{H}_{1}, \ldots, \mathbf{H}_{k}$ are matrices with the same number of columns, $\left[\mathbf{H}_{1} ; \ldots ; \mathbf{H}_{k}\right]$ stands for the matrix with the same number of columns obtained by staking vertically $\mathbf{H}_{1}, \ldots$, and $\mathbf{H}_{k}$.

\section{System Model And PRoblem Formulation}

\section{A. System Model}

We consider a region covered by a macro cellular BS and a set of $M$ small-cell BSs denoted by $\mathcal{M}=\{1,2, \ldots, M\}$. These BSs serve $N$ users denoted by the set $\mathcal{N}=\{1,2, \ldots, N\}$. An example of the considered system model is illustrated in Fig. 1. In addition to the macro cellular BS, user $i$ can possibly receive data from a group of small-cell BSs, represented by the set $\mathcal{M}_{i} \subseteq \mathcal{M} .^{1}$ For notational convenience we refer to the macro BS as BS 0 , and let $\overline{\mathcal{N}}=\mathcal{M} \cup\{0\}$ and $\overline{\mathcal{M}}_{i}=\mathcal{M}_{i} \cup\{0\}$ be the set of all BSs in the entire network and the set of all BSs that can serve user $i$, respectively. Likewise, we define $\mathcal{N}_{j} \subseteq \mathcal{N}$ to be the set of users that can receive data from $\mathrm{BS} j$. The numbers of antennas equipped at BS $j$ and user $i$ are denoted by $K_{j}$ and $L_{i}$, respectively.

\footnotetext{
${ }^{1}$ If a user is only able to receive data from the macro BS then we simply set $\mathcal{M}_{i}=\emptyset$.
} 
The channel between user $i$ and BS $j$ is represented by $\mathbf{H}_{i j} \in$ $\mathbb{C}^{L_{i} \times K_{j}}$. Throughout the paper, perfect channel state information (CSI) is assumed to be available [4]-[6]. For channel acquisition and related signaling overhead, we note that $\mathbf{H}_{i j}$ can be estimated at user $i$ without requiring signaling overhead. In case of frequency division duplex (FDD) mode, user $i$ sends $\mathbf{H}_{i j}$ to $\mathrm{BS} j$ by a control channel, which certainly incurs feedback overhead. In case of time division duplex mode (TDD), given that the channel coherence time is larger than the transmission time, BS $j$ can estimate $\mathbf{H}_{i j}$ by exploiting the channel reciprocity. Thus there is no signaling overhead to acquire $\mathbf{H}_{i j}$ at user $i$ and $\mathrm{BS} j$ in this case. Throughout the paper, we suppose that the cost for the exchange of CSI is negligible compared to that of information data, and thus is not taken into account.

In this paper we assume linear precoding is adopted at all BSs. Particularly, BS $j$ sends a vector of symbols $\mathbf{x}_{i j} \in \mathbb{C}^{L_{i} \times 1}$ to user $i, i \in \mathcal{N}_{j}$, using the precoding matrix $\mathbf{G}_{i j} \in \mathbb{C}^{K_{j} \times L_{i}}$. With these introduced notations, the received signal at user $i$ is given by

$$
\begin{aligned}
\mathbf{y}_{i}=\mathbf{H}_{i 0} \mathbf{G}_{i 0} \mathbf{x}_{i 0} & +\underbrace{\sum_{j \in \mathcal{M}_{i}} \mathbf{H}_{i j} \mathbf{G}_{i j} \mathbf{x}_{i j}}_{\text {from small- cells }} \\
& +\underbrace{\sum_{j \in \overline{\mathcal{M}}_{i}} \sum_{m \in \mathcal{N}_{j}, m \neq i} \mathbf{H}_{i j} \mathbf{G}_{m j} \mathbf{x}_{m j}}_{\text {interference }}+\mathbf{n}_{i}
\end{aligned}
$$

where $\mathbf{n}_{i} \in \mathbb{C}^{L_{i} \times 1}$ is the white complex-Gaussian noise vector with distribution $\mathcal{e} \mathcal{N}\left(0, \sigma_{i}^{2} \mathbf{I}\right)$. The second summation in the right side of (1) represents the desired signals sent from all small-cell BSs associated with user $i$. In this paper we focus on coherent coordination, i.e., all the small-cell BSs in $\mathcal{M}_{i}$ and the macro BS send the same signal to user $i$, i.e., $\mathbf{x}_{i j}=$ $\mathbf{x}_{i}, \forall j \in \overline{\mathcal{M}}_{i}$. However, we remark that the proposed methods can also be extended to noncoherent coordination where a user is also served by multiple BSs, but the intended information is coded and transmitted independently at each BS, i.e. $\mathbf{x}_{i j} \neq \mathbf{x}_{i l}, \forall j \neq l[5]$.

For coherent transmission, the received signal in (1) can be rearranged as

$$
\mathbf{y}_{i}=\left(\sum_{j \in \overline{\mathcal{M}}_{i}} \mathbf{H}_{i j} \mathbf{G}_{i j}\right) \mathbf{x}_{i}+\sum_{m \neq i}\left(\sum_{j \in \overline{\mathcal{M}}_{i}} \mathbf{H}_{i j} \mathbf{G}_{m j}\right) \mathbf{x}_{m}+\mathbf{n}_{i} .
$$

Assuming zero-forcing precoding technique, we can eliminate the interference term by designing $\left\{\mathbf{G}_{m j}\right\}$ such that $\sum_{j \in \overline{\mathcal{M}}_{i}}\left(\mathbf{H}_{i j} \mathbf{G}_{m j}\right)=\mathbf{0}$ for all $m \neq i$. Consequently, the data rate of user $i$ is written as

$$
\begin{aligned}
R_{i}\left(\left\{\mathbf{G}_{i j}\right\}\right) & =\log \left|\mathbf{I}+\frac{1}{\sigma_{i}^{2}}\left(\sum_{j \in \overline{\mathcal{M}}_{i}} \mathbf{H}_{i j} \mathbf{G}_{i j}\right)\left(\sum_{j \in \overline{\mathcal{M}}_{i}} \mathbf{H}_{i j} \mathbf{G}_{i j}\right)^{H}\right| \\
& =\log \left|\mathbf{I}+\mathbf{H}_{i} \mathbf{G}_{i} \mathbf{G}_{i}^{H} \mathbf{H}_{i}^{H}\right|
\end{aligned}
$$

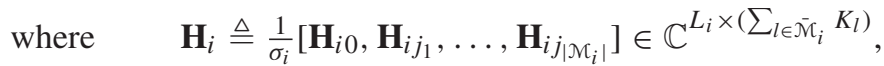
$\mathbf{G}_{i} \triangleq\left[\mathbf{G}_{i 0} ; \mathbf{G}_{i j_{1}} ; \ldots ; \mathbf{G}_{i j_{\left|\mathcal{M}_{i}\right|}}\right] \in \mathbb{C}^{\left(\sum_{l \in \overline{\mathcal{M}}_{i}} K_{l}\right) \times L_{i}}$.

\section{B. Problem Formulation}

To formulate the EEmax problem we briefly present a power consumption model that has been commonly used in the literature. In addition to the data dependent power, this power consumption model also includes the data independent power called circuit power, which is consumed by the circuits operating at BSs and users. Let $p_{j}^{\text {cirTx }}$ and $p_{i}^{\text {cirRx }}$ denote the circuit power of the idle mode of BS $j$ and user $i$, respectively. Let $p_{i j}^{\text {cirCo }}$ denote extra circuit power consumption when BS $j$ transmits data to user $i$ (this circuit power includes the coordinated processing among BSs to transmit data). Then, the total circuit power is given by

$$
P^{\mathrm{cir}}=\sum_{j \in \overline{\mathcal{M}}} p_{j}^{\mathrm{cirTx}}+\sum_{i \in \mathcal{N}} p_{i}^{\mathrm{cirRx}}+\sum_{j \in \overline{\mathcal{M}}} \sum_{i \in \mathcal{N}_{j}} p_{i j}^{\text {cirCo }} .
$$

In summary the total power consumption in the network is given by

$$
P^{\text {Total }}\left(\left\{\mathbf{G}_{i j}\right\}\right)=\sum_{j \in \overline{\mathcal{M}}} \frac{1}{\lambda_{j}} \sum_{i \in \mathcal{N}_{j}} \operatorname{Tr}\left(\mathbf{G}_{i j} \mathbf{G}_{i j}^{H}\right)+P^{\mathrm{cir}}
$$

where $\lambda_{j} \in(0,1)$ is the power efficiency of the amplifier of BS $j$. Now the EEmax problem can be mathematically stated as

$$
\underset{\left\{\mathbf{G}_{i j}\right\}}{\operatorname{maximize}} \frac{\sum_{i \in \mathcal{N}} a_{i} R_{i}\left(\left\{\mathbf{G}_{i j}\right\}\right)}{P^{\text {Total }}\left(\left\{\mathbf{G}_{i j}\right\}\right)}
$$

subject to

$$
\begin{aligned}
& \sum_{j \in \overline{\mathcal{M}}_{i}} \mathbf{H}_{i j} \mathbf{G}_{m j}=\mathbf{0}, \forall m \neq i, i \in \mathcal{N} \\
& R_{i} \geq \bar{R}_{i}, \forall i \in \mathcal{N}, \\
& \sum_{i \in \mathcal{N}_{j}} \operatorname{Tr}\left(\mathbf{G}_{i j} \mathbf{G}_{i j}^{H}\right) \leq P_{j}, \forall j \in \overline{\mathcal{M}}, \\
& \sum_{i \in \mathcal{N}_{j}}\left[\mathbf{G}_{i j} \mathbf{G}_{i j}^{H}\right]_{k, k} \leq P_{j k}, \forall k=1, \ldots, K_{j}, \forall j \in \overline{\mathcal{M}}
\end{aligned}
$$

where $R_{i}\left(\left\{\mathbf{G}_{i j}\right\}\right)$ defined in (3) is the data rate of user $i$. The coefficients $a_{i}$ 's in (6a) are introduced to weight the users' priority. The constraint in (6c) imposes a QoS requirement on user $i$. We consider both types of power constraints at each BS in (6), namely sum power constraint (SPC) in (6d), where $P_{j}$ is the maximum total transmit power for $\mathrm{BS} j$, and per-antenna power constraints in (6e), where $P_{j k}$ is the maximum transmission power of the $k$ th antenna of BS $j$ [23]. In this work, we suppose that a proper scheduler is available such that problem (6) is feasible [24].

\section{Optimal Precoder Design: Centralized And DECENTRALIZED APPROACHES}

To solve the EEmax problem, we first eliminate the zero-interference constraint in (6b) to obtain a simplified formulation. Toward this end let us define the matrix $\quad \overline{\mathbf{H}}_{i j} \triangleq\left[\mathbf{H}_{1 j} ; \mathbf{H}_{2 j} ; \ldots ; \mathbf{H}_{(i-1) j} ; \mathbf{H}_{(i+1) j} ; \ldots ; \mathbf{H}_{N j}\right] \in$ 
$\mathbb{C}^{\left(\sum_{m \neq i} L_{m}\right) \times K_{j}}, j \in \overline{\mathcal{M}}_{i}$, which stacks all the channels from BS $j$ to other users, excluding user $i$, and the matrix $\overline{\mathbf{H}}_{i} \triangleq$ $\left[\overline{\mathbf{H}}_{i 0}, \overline{\mathbf{H}}_{i j_{1}}, \ldots, \overline{\mathbf{H}}_{i j\left|\mathcal{M}_{i}\right|}\right] \in \mathbb{C}^{\left(\sum_{m \neq i} L_{m}\right) \times\left(\sum_{j \in \overline{\mathcal{M}}_{i}} K_{j}\right)}, j_{k} \in \mathcal{M}_{i}$. Then, the zero-interference constraint $(6 \mathrm{~b})$ can be rewritten as $\overline{\mathbf{H}}_{i} \mathbf{G}_{i}=\mathbf{0}$ for all $i \in \mathcal{N}$. In this paper we assume that

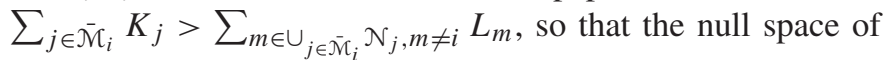
$\overline{\mathbf{H}}_{i}$ exists. The assumption simply means that the total number transmit antennas of all base stations serving user $i$ is larger than the total number of receive antennas of all other users, excluding user $i$. In practice, this assumption can be easily satisfied if the number of transmit antennas at base stations is large or the number of users is small. For a physical interpretation, the assumption requires that we need to have enough spatial diversity (or dimensions) to achieve interference-free transmission to all users.

Now let $\quad \mathbf{B}_{i} \in \mathbb{C}^{\left(\sum_{j \in \overline{\mathcal{M}}_{i}} K_{j}\right) \times \hat{L}_{i}}, \quad \hat{L}_{i}=\sum_{j \in \overline{\mathcal{M}}_{i}} K_{j}-$ $\sum_{m \in \cup_{j \in \overline{\mathcal{N}}_{i}} \mathcal{N}_{j}, m \neq i} L_{m}$, be an orthogonal basis of the null space of $\overline{\mathbf{H}}_{i}$ [17], [24]. Then we can find $\mathbf{G}_{i}$ as $\mathbf{G}_{i}=\mathbf{B}_{i} \tilde{\mathbf{G}}_{i}$, where $\tilde{\mathbf{G}}_{i} \in \mathbb{C}^{\hat{L}_{i} \times L_{i}}$, and write the data rate of user $i$ in (3) as $R_{i}\left(\tilde{\mathbf{G}}_{i}\right)=\log \left|\mathbf{I}+\tilde{\mathbf{H}}_{i} \tilde{\mathbf{G}}_{i} \tilde{\mathbf{G}}_{i}^{H} \tilde{\mathbf{H}}_{i}^{H}\right|$, where $\tilde{\mathbf{H}}_{i} \triangleq \mathbf{H}_{i} \mathbf{B}_{i}$. We note that the rate function $R_{i}\left(\tilde{\mathbf{G}}_{i}\right)$ is neither convex nor concave with $\tilde{\mathbf{G}}_{i}$. A trick commonly used in the literature to overcome this problem is to define new design variables $\boldsymbol{\Psi}_{i} \triangleq \tilde{\mathbf{G}}_{i} \tilde{\mathbf{G}}_{i}^{H} \in \mathbb{C}^{\hat{L}_{i} \times \hat{L}_{i}}$ (see e.g., [9], [25] and references therein). To rewrite problem (6) in terms of $\boldsymbol{\Psi}_{i}$, we define $\mathbf{A}_{i j_{t}} \triangleq\left[\mathbf{0}_{K_{j_{t}} \times\left(\sum_{m=0}^{t-1} K_{l_{m}}\right)}, \mathbf{I}_{K_{j_{t}}}, \mathbf{0}_{K_{j_{t}} \times\left(\sum_{m=t+1}^{\left|\mathcal{M}_{i}\right|} K_{l_{m}}\right)}\right]$ for BS $j_{t} \in$ $\overline{\mathcal{M}}_{i}$ and note that $\operatorname{Tr}\left(\mathbf{G}_{i j_{t}} \mathbf{G}_{i j_{t}}^{H}\right)=\operatorname{Tr}\left(\mathbf{A}_{i j_{t}} \mathbf{B}_{i} \tilde{\mathbf{G}}_{i} \tilde{\mathbf{G}}_{i}^{H} \mathbf{B}_{i}^{H} \mathbf{A}_{i j_{t}}^{H}\right)=$ $\operatorname{Tr}\left(\tilde{\mathbf{B}}_{i j_{t}} \boldsymbol{\Psi}_{i} \tilde{\mathbf{B}}_{i, j_{t}}^{H}\right)$ where $\tilde{\mathbf{B}}_{i j_{t}} \triangleq \mathbf{A}_{i j_{t}} \mathbf{B}_{i}$. With these notations, problem (6) is equivalently reformulated as

$$
\begin{array}{cc}
\operatorname{maximize}_{\left\{\boldsymbol{\Psi}_{i} \succeq \mathbf{0}\right\}} & \frac{\sum_{i \in \mathcal{N}} a_{i} \log \left|\mathbf{I}+\tilde{\mathbf{H}}_{i} \boldsymbol{\Psi}_{i} \tilde{\mathbf{H}}_{i}^{H}\right|}{\sum_{j \in \overline{\mathcal{N}}} \frac{1}{\lambda_{j}} \sum_{i \in \mathcal{N}_{j}} \operatorname{Tr}\left(\tilde{\mathbf{B}}_{i j} \boldsymbol{\Psi}_{i} \tilde{\mathbf{B}}_{i j}^{H}\right)+P^{\mathrm{cir}}} \\
\text { subject to } & \log \left|\mathbf{I}+\tilde{\mathbf{H}}_{i} \boldsymbol{\Psi}_{i} \tilde{\mathbf{H}}_{i}^{H}\right| \geq \bar{R}_{i}, \forall i, \\
& \sum_{i \in \mathcal{N}_{j}} \operatorname{Tr}\left(\tilde{\mathbf{B}}_{i j} \boldsymbol{\Psi}_{i} \tilde{\mathbf{B}}_{i, j}^{H}\right) \leq P_{j}, \forall j, \\
& \sum_{i \in \mathcal{N}_{j}}\left[\tilde{\mathbf{B}}_{i j} \boldsymbol{\Psi}_{i} \tilde{\mathbf{B}}_{i j}^{H}\right]_{k, k} \leq P_{j k}, \forall k, j
\end{array}
$$

We note that the rank constraints $\operatorname{rank}\left(\boldsymbol{\Psi}_{i}\right) \leq L_{i}$, which are necessary to recover $\tilde{\mathbf{G}}_{i}$ from $\boldsymbol{\Psi}_{i}$, have been implicitly omitted in (7) for a tractable reason. Fortunately, we show in Section IIIA1 that the rank constraints are satisfied for optimal solutions of (7). Specifically, we will prove that if $\left\{\Psi_{i}^{*}\right\}$ is optimal to (7) then $\operatorname{rank}\left(\boldsymbol{\Psi}_{i}^{*}\right) \leq \min \left(L_{i}, \hat{L}_{i}\right)$.

We recall that the function $\log \left|\mathbf{I}+\tilde{\mathbf{H}}_{i} \boldsymbol{\Psi}_{i} \tilde{\mathbf{H}}_{i}^{H}\right|$ is concave with $\boldsymbol{\Psi}_{i}$ on the domain $\boldsymbol{\Psi}_{i} \succeq \mathbf{0}$ [26]. Thus, all the constraints in (7) are convex and the objective in (7a) is a ratio between a concave and convex function. That is to say, (7) is a concaveconvex fractional program. In the following we present both centralized and decentralized methods to solve (7) optimally.
Algorithm 1. The proposed centralized algorithm to solve (7) based on Dinkelbach's method

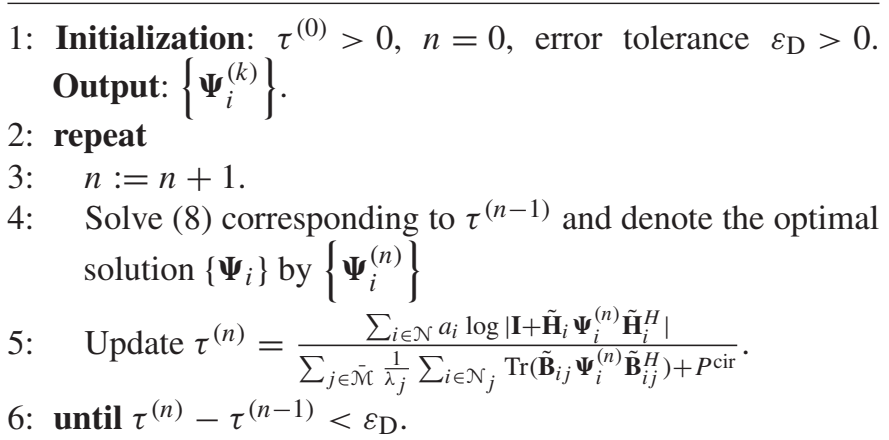

\section{A. Centralized Approach: Dinkelbach's Method}

A well-known method to solve concave-convex fractional problems such as the one in (7) is based on Dinkelbach's method [27], in which a series of so-called parameterized convex problems are solved until convergence. In this subsection, we particularize the Dinkelbach method to find optimal solutions of (7), and provide insights into the structure of the solution. Let us introduce the following parameterized subproblem

$$
\begin{aligned}
\underset{\left\{\boldsymbol{\Psi}_{i} \succeq \mathbf{0}\right\}}{\operatorname{maximize}} & \sum_{i \in \mathcal{N}} a_{i} \log \left|\mathbf{I}+\tilde{\mathbf{H}}_{i} \boldsymbol{\Psi}_{i} \tilde{\mathbf{H}}_{i}^{H}\right| \\
& -\tau\left(\sum_{j \in \overline{\mathcal{M}}} \frac{1}{\lambda_{j}} \sum_{i \in \mathcal{N}_{j}} \operatorname{Tr}\left(\tilde{\mathbf{B}}_{i j} \boldsymbol{\Psi}_{i} \tilde{\mathbf{B}}_{i j}^{H}\right)+P^{\mathrm{cir}}\right)
\end{aligned}
$$

subject to $(7 b),(7 c),(7 d)$

for a given parameter $\tau>0$. We note that (8) is a convex program for a fixed $\tau$, and thus can be solved efficiently. To solve (7) by Dinkelbach's method requires an iterative procedure where subproblem (8) is solved and parameter $\tau$ is updated in each iteration. Before presenting a method to solve (8), we first outline the proposed design based on the Dinkelbach method to solve the EEmax problem in Algorithm 1. The convergence analysis of Algorithm 1 was studied in [27], which is skipped here due to space limitation.

1) Solving Subproblem (8) Using Closed-Form Expressions: Exploiting the problem specifics, we now present a way to solve (8) using closed-formed expressions. We further assume that the EEmax problem is strictly feasible, i.e., there exists $\left\{\boldsymbol{\Psi}_{i}\right\}$ such that all the constraints in (7) are satisfied with strict inequality. Accordingly, the duality gap of the convex program in (8) is zero and its optimal solutions can be found by solving the dual problem [26]. To this end, let us form the partial Lagrangian function of (8), which is given by

$$
\begin{aligned}
& \mathcal{L}_{\tau}\left(\left\{\boldsymbol{\Psi}_{i}\right\},\left\{\eta_{i}\right\},\left\{\mu_{j}\right\},\left\{\xi_{j k}\right\}\right)=\sum_{i \in \mathcal{N}} a_{i} \log \left|\mathbf{I}+\tilde{\mathbf{H}}_{i} \boldsymbol{\Psi}_{i} \tilde{\mathbf{H}}_{i}^{H}\right| \\
& -\tau\left(\sum_{j \in \overline{\mathcal{M}}} \frac{1}{\lambda_{j}} \sum_{i \in \mathcal{N}_{j}} \operatorname{Tr}\left(\check{\mathbf{B}}_{i j} \boldsymbol{\Psi}_{i}\right)\right)
\end{aligned}
$$




$$
\begin{aligned}
& +\sum_{i \in \mathcal{N}} \eta_{i}\left(\log \left|\mathbf{I}+\tilde{\mathbf{H}}_{i} \boldsymbol{\Psi}_{i} \tilde{\mathbf{H}}_{i}^{H}\right|-\bar{R}_{i}\right) \\
& +\sum_{j \in \overline{\mathcal{M}}} \mu_{j}\left(P_{j}-\sum_{i \in \mathcal{N}_{j}} \operatorname{Tr}\left(\check{\mathbf{B}}_{i j} \boldsymbol{\Psi}_{i}\right)\right) \\
& +\sum_{j \in \overline{\mathcal{M}}} \sum_{k=1}^{K_{j}} \xi_{j k}\left(P_{j k}-\sum_{i \in \mathcal{N}_{j}} \operatorname{Tr}\left(\hat{\mathbf{B}}_{i j k} \boldsymbol{\Psi}_{i}\right)\right)
\end{aligned}
$$

where $\check{\mathbf{B}}_{i j} \triangleq \tilde{\mathbf{B}}_{i j}^{H} \tilde{\mathbf{B}}_{i j}, \quad \dot{\mathbf{B}}_{i j k}=\left[\begin{array}{lll}\mathbf{0}_{k-1}^{T} & 1 & \mathbf{0}_{K_{j}-k}^{T}\end{array}\right] \tilde{\mathbf{B}}_{i j}$ and $\hat{\mathbf{B}}_{i j k} \triangleq$ $\dot{\mathbf{B}}_{i j k}^{H} \dot{\mathbf{B}}_{i j k} ;\left\{\eta_{i}\right\}_{i \in \mathcal{N}},\left\{\mu_{j}\right\}_{j \in \overline{\mathcal{M}}}$, and $\left\{\xi_{j k}\right\}_{j \in \overline{\mathcal{M}}, k=1, \ldots, K_{j}}$ are Lagrangian multipliers corresponding to $(7 \mathrm{~b}),(7 \mathrm{c})$, and $(7 \mathrm{~d})$, respectively. We have ignored the term $\tau P^{\text {cir }}$ in (9) since it is a constant, and thus has no effect on finding optimal solutions. In the dual method, we first need to find the dual function which is given by the following problem

$$
\begin{aligned}
\mathcal{D}\left(\left\{\eta_{i}\right\},\left\{\mu_{j}\right\},\left\{\xi_{j k}\right\}\right) & \\
& =\operatorname{maximize}_{\left\{\boldsymbol{\Psi}_{i} \succeq \mathbf{0}\right\}} \mathcal{L}_{\tau}\left(\left\{\boldsymbol{\Psi}_{i}\right\},\left\{\eta_{i}\right\},\left\{\mu_{j}\right\},\left\{\xi_{j k}\right\}\right) .
\end{aligned}
$$

To tackle the maximization in (10) for a given set of multipliers $\left\{\eta_{i}\right\},\left\{\mu_{j}\right\}$, and $\left\{\xi_{j k}\right\}$, we rewrite the Lagrangian function as

$$
\begin{aligned}
& \mathcal{L}_{\tau}\left(\left\{\boldsymbol{\Psi}_{i}\right\},\left\{\eta_{i}\right\},\left\{\mu_{j}\right\},\left\{\xi_{j k}\right\}\right) \\
& =\sum_{i \in \mathcal{N}}\left(\left(a_{i}+\eta_{i}\right) \log \left|\mathbf{I}+\tilde{\mathbf{H}}_{i} \boldsymbol{\Psi}_{i} \tilde{\mathbf{H}}_{i}^{H}\right|-\operatorname{Tr}\left(\ddot{\mathbf{B}}_{i} \boldsymbol{\Psi}_{i}\right)-\eta_{i} \bar{R}_{i}\right) \\
& \quad+\sum_{j \in \overline{\mathcal{M}}}\left(\mu_{j} P_{j}+\sum_{k=1}^{K_{j}} \xi_{j k} P_{j k}\right) \\
& =\sum_{i \in \mathcal{N}}\left(\left(a_{i}+\eta_{i}\right) \log \left|\mathbf{I}+\dot{\mathbf{H}}_{i} \overline{\boldsymbol{\Psi}}_{i} \dot{\mathbf{H}}_{i}^{H}\right|-\operatorname{Tr}\left(\overline{\mathbf{\Psi}}_{i}\right)-\eta_{i} \bar{R}_{i}\right) \\
& \quad+\sum_{j \in \overline{\mathcal{M}}}\left(\mu_{j} P_{j}+\sum_{k=1}^{K_{j}} \xi_{j k} P_{j k}\right)
\end{aligned}
$$

where $\ddot{\mathbf{B}}_{i}=\sum_{j \in \overline{\mathcal{M}}_{i}}\left(\left(\frac{\tau}{\lambda_{j}}+\mu_{j}\right) \check{\mathbf{B}}_{i j}+\sum_{k=1}^{K_{j}} \xi_{j k} \hat{\mathbf{B}}_{i j k}\right), \quad \overline{\boldsymbol{\Psi}}_{i}=$ $\ddot{\mathbf{B}}_{i}^{1 / 2} \boldsymbol{\Psi}_{i} \ddot{\mathbf{B}}_{i}^{1 / 2}$ and $\dot{\mathbf{H}}_{i}=\tilde{\mathbf{H}}_{i} \ddot{\mathbf{B}}_{i}^{-1 / 2}$. We note that $\ddot{\mathbf{B}}_{i}$ is invertible for all $\tau>0 .^{2}$ To see this, let us rewrite $\ddot{\mathbf{B}}_{i}$ such as $\ddot{\mathbf{B}}_{i}=\mathbf{B}_{i}^{H} \tilde{\mathbf{A}}_{i} \mathbf{B}_{i}$ where $\tilde{\mathbf{A}}_{i}=\sum_{j \in \overline{\mathcal{M}}_{i}} \mathbf{A}_{i j}^{H} \operatorname{diag}\left(\frac{\tau}{\lambda_{j}}+\mu_{j}+\right.$ $\left.\xi_{j 1}, \ldots, \frac{\tau}{\lambda_{j}}+\mu_{j}+\xi_{j K_{j}}\right) \mathbf{A}_{i j}$. We note that $\tilde{\mathbf{A}}_{i}$ is actually a diagonal matrix of positive diagonal entries for $\tau>0$, and thus $\ddot{\mathbf{B}}_{i}$ is invertible. Let the SVD of $\dot{\mathbf{H}}_{i}$ as $\dot{\mathbf{H}}_{i}=\mathbf{U}_{i} \boldsymbol{\Xi}_{i} \mathbf{V}_{i}^{H}$, where $\mathbf{\Xi}_{i}=\operatorname{diag}\left(\sigma_{i 1}, \ldots, \sigma_{i \tilde{L}_{i}}\right), \tilde{L}_{i} \triangleq \min \left(L_{i}, \hat{L}_{i}\right)$, then the optimal solution to (10) is given by [9], [28]

$$
\boldsymbol{\Psi}_{i}^{*}=\ddot{\mathbf{B}}_{i}^{-1 / 2} \mathbf{V}_{i} \mathbf{D}_{i} \mathbf{V}_{i}^{H} \ddot{\mathbf{B}}_{i}^{-1 / 2}
$$

\footnotetext{
${ }^{2}$ In fact, $\ddot{\mathbf{B}}_{i}$ is also invertible for $\tau=0$. However, we omit this case here since, in Algorithm 1, we can always find $\tau^{(0)}>0$.
}

where $\quad \mathbf{D}_{i}=\operatorname{diag}\left(\left[\left(a_{i}+\eta_{i}\right)-1 / \sigma_{i 1}^{2}\right]^{+}, \ldots,\left[\left(a_{i}+\eta_{i}\right)-\right.\right.$ $\left.\left.1 / \sigma_{i \tilde{L}_{i}}^{2}\right]^{+}\right)$, and $[x]^{+}=\max (0, x)$. Since optimal solutions to (8) for given $\tau$ admit the structure in (12), it immediately follows that optimal solutions to (7) always satisfy the rank constraints, i.e., $\operatorname{rank}\left(\boldsymbol{\Psi}_{i}^{*}\right) \leq \tilde{L}_{i}$. Moreover, looking at (12) we can see that optimal solutions are obtained from the waterfilling algorithm with a fixed water level, which is partially determined by $\tau$.

The final step in the dual method is to find optimal Lagrangian multipliers, which are obtained by solving the following dual problem

$$
\underset{\left\{\eta_{i}\right\} \geq \mathbf{0},\left\{\mu_{j}\right\} \geq \mathbf{0},\left\{\xi_{j k}\right\} \geq \mathbf{0}}{\operatorname{minimize}} \mathcal{D}\left(\left\{\eta_{i}\right\},\left\{\mu_{j}\right\},\left\{\xi_{j k}\right\}\right) .
$$

Since $\mathcal{D}\left(\left\{\eta_{i}\right\},\left\{\mu_{j}\right\},\left\{\xi_{j k}\right\}\right)$ is not necessarily differentiable, we employ subgradient method to obtain an optimal solution of (13). In this way, solution of (8) is obtained after $\left\{\boldsymbol{\Psi}_{i}\right\}$, $\left\{\eta_{i}\right\},\left\{\mu_{j}\right\}$, and $\left\{\xi_{j k}\right\}$ are iteratively updated until convergence. Specifically, the Lagrange multiplier variables are updated at the $(\theta+1)$ th iteration as follows

$$
\begin{aligned}
& \eta_{i}^{(\theta+1)}=\left[\eta_{i}^{(\theta)}-\kappa^{(\theta)}\left(\log \left|\mathbf{I}+\tilde{\mathbf{H}}_{i} \Psi_{i}^{(\theta+1)} \tilde{\mathbf{H}}_{i}^{H}\right|-\bar{R}_{i}\right)\right]^{+} \\
& \mu_{j}^{(\theta+1)}=\left[\mu_{j}^{(\theta)}-\kappa^{(\theta)}\left(P_{j}-\sum_{i \in \mathcal{N}_{j}} \operatorname{Tr}\left(\check{\mathbf{B}}_{i j} \Psi_{i}^{(\theta+1)}\right)\right)\right]^{+} \\
& \xi_{j k}^{(\theta+1)}=\left[\xi_{j k}^{(\theta)}-\kappa^{(\theta)}\left(P_{j k}-\sum_{i \in \mathcal{N}_{j}} \operatorname{Tr}\left(\hat{\mathbf{B}}_{i j k} \Psi_{i}^{(\theta+1)}\right)\right)\right]^{+}
\end{aligned}
$$

where $\boldsymbol{\Psi}_{i}^{(\theta+1)}$ is given in (12) and $\kappa^{(\theta)}>0$ is the step size. Subgradient method is guaranteed to converge if the step size is chosen properly. In this paper we use a diminishing step size rule, i.e. $\kappa^{(\theta)}=b / \sqrt{\theta}$ where $b$ is constant [29].

2) Solving (8) Using Interior-Point Convex Solvers: Solving (8) by the dual method offers useful insights into the EEmax problem as discussed above. However, the subgradient method for solving the dual problem presented above is commonly known to suffer from very slow convergence, especially for large scale networks (i.e., large numbers of BSs and/or transmit antennas). On the other hand, there are many efficient convex programming solvers based on interior-point methods, which generally show superior convergence results, compared to subgradient methods [26]. Unfortunately, to the best of our knowledge, no off-the-shelf convex solver can solve (8) at its current form. Thus, it is necessary to transform (8) into a more standard form, to avail of these powerful modern convex solvers. For this purpose, we first introduce the following lemma which reformulates (8) as a MAXDET program [30]. 
Lemma 1: Problem (8) is equivalent to the following convex problem

$$
\underset{\left\{\Psi_{i}\right\},\left\{\boldsymbol{\Lambda}_{i}\right\}}{\operatorname{maximize}} \sum_{i \in \mathcal{N}} a_{i} \sum_{l=1}^{L_{i}} \log q_{i l}-\tau P^{\text {Total }}
$$

subject to

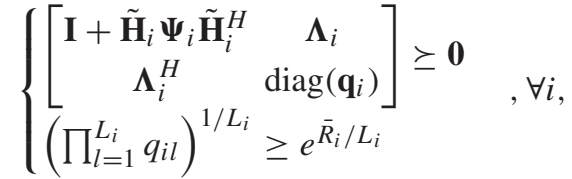

$\boldsymbol{\Lambda}_{i}: L_{i} \times L_{i}$ lower triangular matrix; $\Psi_{i} \succeq \mathbf{0}, \forall i$,

$$
\text { (7c), (7d) }
$$

where auxiliary variable matrices $\left\{\boldsymbol{\Lambda}_{i}\right\}$ are newly introduced, and $\mathbf{q}_{i} \triangleq\left\{q_{i l}\right\}_{l}=\operatorname{diag}\left(\boldsymbol{\Lambda}_{i}\right)$ is the vector of diagonal entries of $\Lambda_{i}$.

Proof: The proof is due to a result in [31, Section 4.2] and is provided in the Appendix.

We note that (15) is indeed a MAXDET problem since the constraint in the form of $\left(\prod_{i=1}^{n} x_{i}\right)^{1 / n} \geq t$ (like the one in (15b) can be represented by a series of at most $\left\lceil\log _{2} n\right\rceil$ linear matrix inequalities (LMIs). For example when $n=4$, we have

$$
\left(x_{1} x_{2} x_{3} x_{4}\right)^{1 / 4} \geq t \Leftrightarrow x_{1} x_{2} \geq t_{1}^{2} ; x_{3} x_{4} \geq t_{2}^{2} ; t_{1} t_{2} \geq t^{2} .
$$

Recall that the constraint $x y \geq z^{2}$ is called a rotated cone, which is equivalent to the LMI $\left[\begin{array}{l}x \\ z \\ z\end{array}\right] \succeq \mathbf{0}$. We remark that a dedicated solver for MAXDET problems is SDPT3 [32].

\section{B. Distributed Approach}

The EEmax problem can be solved in a centralized manner as presented in the preceding section, however, for practical implementation, decentralized algorithms are of more interest. Our purpose in this subsection is to propose a distributed approach to solve (7) optimally. Building distributed implementations based on the Dinkelbach method is probably not a good choice, although subproblem (8) can be solved distributedly using the dual decomposition method, which is shown in (14). The main reason is that updating $\tau$ (i.e., line 5 of Algorithm 1) still requires a central node which collects information about data rate and power from all base stations and users. To facilitate a distributed solution, we first reformulate the EEmax problem as a convex program using a parameter-free method which eliminates the need for an outer loop as in Dinkelbach's method.

1) An Equivalent Parameter-Free Formulation: By Lemma 1, we can equivalently rewrite the EEmax problem as

$$
\begin{aligned}
& \underset{\left\{\boldsymbol{\Psi}_{i}\right\},\left\{\mathbf{\Lambda}_{i}\right\}}{\operatorname{maximize}} \frac{\sum_{i \in \mathcal{N}} a_{i} \sum_{l=1}^{L_{i}} \log q_{i l}}{\sum_{j \in \overline{\mathcal{M}}} \frac{1}{\lambda_{j}} \sum_{i \in \mathcal{N}_{j}} \operatorname{Tr}\left(\tilde{\mathbf{B}}_{i j} \Psi_{i} \tilde{\mathbf{B}}_{i j}^{H}\right)+P^{\text {cir }}} \\
& \text { subject to (15b), (15c), (15d). }
\end{aligned}
$$

The key to arriving at a parameter-free formulation of (17) is to make use of the Charnes-Cooper transformation [19].
Specifically, let us introduce $\boldsymbol{\Psi}_{i}=\tilde{\boldsymbol{\Psi}}_{i} / \phi$ and $\boldsymbol{\Lambda}_{i}=\tilde{\boldsymbol{\Lambda}}_{i} / \phi$ for $\phi>0$. Then, (17) is equivalent to the following convex program

$$
\operatorname{maximize}_{\left\{\tilde{\boldsymbol{\Psi}}_{i}\right\}, \phi,\left\{\tilde{\mathbf{\Lambda}}_{i}\right\}} \sum_{i \in \mathcal{N}} a_{i} \sum_{l=1}^{L_{i}} \phi \log \left(\tilde{q}_{i l} / \phi\right)
$$

subject to

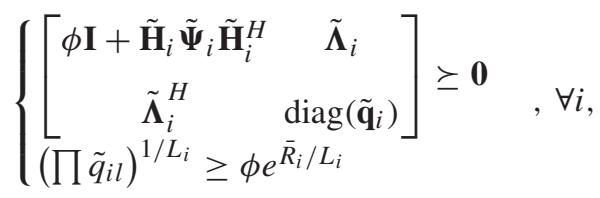

$\tilde{\mathbf{\Lambda}}_{i}: L_{i} \times L_{i}$ lower triangular matrix; $\tilde{\mathbf{\Psi}}_{i} \succeq \mathbf{0}, \forall i$,

$$
\begin{aligned}
& \sum_{j \in \mathcal{\mathcal { M }}} \frac{1}{\lambda_{j}} \sum_{i \in \mathcal{N}_{j}} \operatorname{Tr}\left(\tilde{\mathbf{B}}_{i j} \tilde{\Psi}_{i} \tilde{\mathbf{B}}_{i j}^{H}\right)+\phi P^{\mathrm{cir}}=1, \\
& \sum_{i \in \mathcal{N}_{j}} \operatorname{Tr}\left(\tilde{\mathbf{B}}_{i j} \tilde{\mathbf{\Psi}}_{i} \tilde{\mathbf{B}}_{i j}^{H}\right) \leq \phi P_{j}, \quad \forall j, \\
& \sum_{i \in \mathcal{N}_{j}}\left[\tilde{\mathbf{B}}_{i j} \tilde{\boldsymbol{\Psi}}_{i} \tilde{\mathbf{B}}_{i j}^{H}\right]_{k, k} \leq \phi P_{j k}, \forall k, j .
\end{aligned}
$$

where $\tilde{\mathbf{q}}_{i} \triangleq\left\{\tilde{q}_{i l}\right\}_{l}=\operatorname{diag}\left(\tilde{\mathbf{\Lambda}}_{i}\right)$ is the vector of diagonal entries of $\tilde{\boldsymbol{\Lambda}}_{i}$. The equivalence between (17) and (18) can be briefly proved as follows. Let $\mathcal{R}$ and $\tilde{\mathcal{R}}$ be feasible convex sets of (17) and (18), respectively. Consider the mapping from $\mathcal{R}$ to $\tilde{\mathcal{R}}$ as $\tilde{\boldsymbol{\Psi}}_{i}=\boldsymbol{\Psi}_{i} / P^{\text {Total }}, \tilde{\boldsymbol{\Lambda}}_{i}=\boldsymbol{\Lambda}_{i} / P^{\text {Total }}$, and $\phi=1 / P^{\text {Total }}$ (note that we always have $P^{\text {Total }}>0$ ). It is trivial to check that if $\left(\left\{\boldsymbol{\Psi}_{i}\right\},\left\{\boldsymbol{\Lambda}_{i}\right\}\right) \in \mathcal{R}$, then $\left(\left\{\tilde{\boldsymbol{\Psi}}_{i}\right\}, \phi,\left\{\tilde{\mathbf{\Lambda}}_{i}\right\}\right) \in \tilde{\mathcal{R}}$ with the same objective. Conversely, if $\left(\left\{\tilde{\boldsymbol{\Psi}}_{i}\right\}, \phi,\left\{\tilde{\boldsymbol{\Lambda}}_{i}\right\}\right) \in \tilde{\mathcal{R}}$ then $\phi>0$. Thus, the mapping $\left(\left\{\tilde{\boldsymbol{\Psi}}_{i}\right\}, \phi,\left\{\tilde{\mathbf{\Lambda}}_{i}\right\}\right) \in \tilde{\mathcal{R}} \rightarrow\left(\left\{\tilde{\boldsymbol{\Psi}}_{i} / \phi\right\},\left\{\tilde{\mathbf{\Lambda}}_{i} / \phi\right\}\right) \in$ $\mathcal{R}$ is defined and also yields the same objective. In other words, Charnes-Cooper's transformation is a one-to-one mapping between $\mathcal{R}$ and $\tilde{\mathcal{R}}$ with the same objective. As a result, we can obtain the optimal solution of the EEmax problem by solving the convex problem (18). More importantly, the formulation (18) lends itself to a distributed optimal algorithm which is presented next. Before presenting the proposed distributed algorithm we note that it is the most convenient to derive $\tilde{\mathbf{H}}_{i}$ at user $i$. Specifically, first, user $i$ gathers $\overline{\mathbf{H}}_{i j}$ from BS $j$, for all $j \in \overline{\mathcal{M}}_{i}$, to form $\overline{\mathbf{H}}_{i}$. Then, it derives $\mathbf{B}_{i}$ from the nullspace of $\overline{\mathbf{H}}_{i}$. Finally, it straightforwardly obtains $\tilde{\mathbf{H}}_{i}$ from $\mathbf{B}_{i}$ and $\mathbf{H}_{i}$.

2) ADMM-Based Algorithm: Our goal in this subsection is to develop a distributed algorithm based on (18) to solve the EEmax problem. It is worth pointing out that while the dual decomposion method has been widely considered for distributed solutions for various design problems in wireless communications [33], it cannot be applied to (18) due to the lack of strict convexity. More specifically, the dual function for (18) will not be defined due to the introduced linearity with respect to $\left\{\tilde{\boldsymbol{\Psi}}_{i}\right\}$ in (18), [20]. To overcome this shortcoming, we resort to the ADMM in this paper [20]. It has been generally shown that the ADMM converges quickly, even for the 
large scale networks, making it superior to dual decomposition methods [20].

To begin, we first transform (18) into a form which is more amenable to applying ADMM. Consider an equivalent problem of (18) given by

$$
\underset{\substack{\left\{\tilde{\Psi}_{i}\right\},\left\{\tilde{\boldsymbol{\Lambda}}_{i}\right\},\left\{s_{i j k}\right\}, \phi,\left\{\hat{\phi}_{i}\right\},\left\{\tilde{\phi}_{j}\right\},\left\{v_{i j k}\right\},\left\{v_{i j k}^{\prime}\right\}}}{\operatorname{maximize}} \sum_{i \in \mathcal{N}} a_{i} \sum_{l=1}^{L_{i}} \hat{\phi}_{i} \log \left(\tilde{q}_{i l} / \hat{\phi}_{i}\right)
$$

subject to

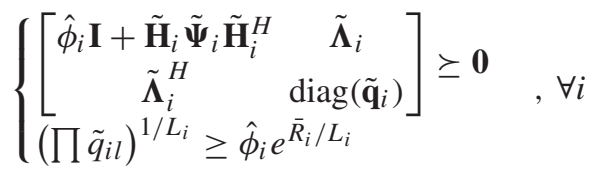

$\tilde{\boldsymbol{\Lambda}}_{i}: L_{i} \times L_{i}$ lower triangular matrix; $\tilde{\mathbf{\Psi}}_{i} \succeq \mathbf{0}, \forall i$,

$$
\begin{aligned}
& \sum_{j \in \overline{\mathcal{M}}} \frac{1}{\lambda_{j}} \sum_{i \in \mathcal{N}_{j}} \sum_{k=1}^{K_{j}} v_{i j k}+\phi P^{\mathrm{cir}}=1 \\
& \hat{\phi}_{i}=\phi, \forall i \in \mathcal{N} ; \tilde{\phi}_{j}=\phi, \forall j \in \overline{\mathcal{M}}, \\
& \operatorname{Tr}\left(\dot{\mathbf{B}}_{i j k} \tilde{\boldsymbol{\Psi}}_{i} \dot{\mathbf{B}}_{i j k}^{H}\right) \leq v_{i j k}^{\prime}, \forall i, j, k \\
& v_{i j k}^{\prime}=s_{i j k}, \quad v_{i j k}=s_{i j k}, \quad \forall i, j, k \\
& \sum_{i \in \mathcal{N}_{j}} \sum_{k=1}^{K_{j}} v_{i j k} \leq \tilde{\phi}_{j} P_{j}, \forall j ; \sum_{i \in \mathcal{N}_{j}} v_{i j k} \leq \tilde{\phi}_{j} P_{j k}, \forall j, k
\end{aligned}
$$

where auxiliary variables are newly introduced in (19) to make it more decomposable. Explicitly, $\left\{v_{i j k}\right\}_{j \in \overline{\mathcal{N}}, i \in \mathcal{N}_{j}, k=1, \ldots, K_{j}}$, $\left\{v_{i j k}^{\prime}\right\}_{i \in \mathcal{N}, j \in \overline{\mathcal{M}}_{i}, k=1, \ldots, K_{j}}$ can be interpreted as the power of the $k$ th antenna that $\mathrm{BS} j$ allocates to user $i$, but locally stored by BSs and users, respectively. In this regard, $s_{i j k}$ 's are called global variables which are introduced to keep the local versions equal by the equalities in $(19 \mathrm{~g})$. Similarly, the purpose of introducing variables $\left\{\hat{\phi}_{i}\right\}_{i \in \mathcal{N}},\left\{\tilde{\phi}_{j}\right\}_{j \in \overline{\mathcal{M}}}$ is to handle the constraints (19b) locally at users, and (19h) locally at BSs. Further, let us define the local feasible set of user $i$ as

$$
\mathcal{O}_{i}=\left\{\tilde{\boldsymbol{\Psi}}_{i}, \tilde{\boldsymbol{\Lambda}}_{i}, \hat{\phi}_{i}, \mathbf{v}_{i}^{\prime} \mid(19 \mathrm{~b}),(19 \mathrm{c}),(19 \mathrm{f})\right\}
$$

where $\mathbf{v}_{i}^{\prime}=\left\{v_{i j k}^{\prime}\right\}_{j \in \overline{\mathcal{M}}_{i}, k=1, \ldots, K_{j}}$, and the local feasible set of BS $j$ as

$$
\tilde{\mathcal{O}}_{j}=\left\{\mathbf{v}_{j}, \tilde{\phi}_{j} \mid(19 \mathrm{~h})\right\}
$$

where $\mathbf{v}_{j}=\left\{v_{i j k}\right\}_{i \in \mathcal{N}_{j}, k=1, \ldots, K_{j}}$. We also define the cost function $f_{i}\left(\hat{\phi}_{i}, \tilde{\boldsymbol{\Lambda}}_{i}\right)=-a_{i} \sum_{l=1}^{L_{i}} \hat{\phi}_{i} \log \left(\tilde{q}_{i l} / \hat{\phi}_{i}\right)$ at user $i$. Let $\boldsymbol{\pi}_{j}=\left[\tilde{\phi}_{j}, \mathbf{v}_{j}^{T}\right]^{T}, \quad \boldsymbol{\pi}_{i}^{\prime}=\left[\hat{\phi}_{i}, \mathbf{v}_{i}{ }^{\prime T}\right]^{T}, \quad \boldsymbol{\alpha}_{j}=\left[\phi, \mathbf{s}_{j}^{T}\right]^{T}$ and $\boldsymbol{\alpha}_{i}^{\prime}=\left[\phi, \mathbf{s}_{i}{ }^{T}\right]^{T}$, where $\mathbf{s}_{j}=\left\{s_{i j k}\right\}_{i \in \mathcal{N}_{j}, k=1, \ldots, K_{j}}$ and $\mathbf{s}_{i}^{\prime}=$ $\left\{s_{i j k}\right\}_{j \in \overline{\mathcal{M}}_{i}, k=1, \ldots, K_{j}}$. With these notations, problem (19) is rewritten as

$$
\underset{\substack{\left\{\tilde{\boldsymbol{\Psi}}_{i}\right\},\left\{\tilde{\boldsymbol{\Lambda}}_{i}\right\},\left\{\boldsymbol{\pi}_{j}\right\},\left\{\boldsymbol{\pi}_{i}^{\prime}\right\},\left\{\boldsymbol{\alpha}_{j}\right\}, \mathbf{t}}}{\operatorname{minimize}} \sum_{i \in \mathcal{N}} f_{i}\left(\hat{\phi}_{i}, \tilde{\boldsymbol{\Lambda}}_{i}\right)
$$

$$
\begin{gathered}
\text { subject to } \mathbf{b}_{j}^{T} \boldsymbol{\pi}_{j}-\frac{1}{M+1}=t_{j}, \forall j \in \overline{\mathcal{M}} ; \sum_{j \in \overline{\mathcal{M}}} t_{j}=0 \\
\pi_{j}=\boldsymbol{\alpha}_{j}, \forall j \in \overline{\mathcal{M}} \\
\boldsymbol{\pi}_{i}^{\prime}=\boldsymbol{\alpha}_{i}^{\prime}, \forall i \in \mathcal{N} \\
\left(\tilde{\boldsymbol{\Psi}}_{i}, \tilde{\boldsymbol{\Lambda}}_{i}, \boldsymbol{\pi}_{i}^{\prime}\right) \in \mathcal{O}_{i}, \forall i \\
\boldsymbol{\pi}_{j} \in \tilde{\mathcal{O}}_{j}, \forall j
\end{gathered}
$$

where $\mathbf{t} \triangleq\left\{t_{j}\right\}_{j \in \overline{\mathcal{M}}}$ are newly introduced variables, and $\mathbf{b}_{j} \triangleq$ $\left[P^{\mathrm{cir}} /(M+1), \mathbf{1}^{T} / \lambda_{j}\right]^{T}$ in $(22 \mathrm{~b})$.

We are now in a position to present the particularized ADMM to solve problem (22). First, we form the partial augmented Lagrangian function of (22) which is given by

$$
\begin{aligned}
\mathcal{L}_{A}( & \left.\left\{\tilde{\boldsymbol{\Lambda}}_{i}\right\},\left\{\boldsymbol{\pi}_{i}^{\prime}\right\},\left\{\boldsymbol{\pi}_{j}\right\},\left\{\boldsymbol{\alpha}_{j}\right\}, \mathbf{t},\left\{\zeta_{i}\right\},\left\{\psi_{j}\right\},\left\{\boldsymbol{\delta}_{j}\right\}, \beta\right) \\
= & \sum_{i \in \mathcal{N}}\left[f_{i}\left(\hat{\phi}_{i}, \tilde{\boldsymbol{\Lambda}}_{i}\right)+\zeta_{i}^{T}\left(\boldsymbol{\pi}_{i}^{\prime}-\boldsymbol{\alpha}_{i}^{\prime}\right)+\frac{c}{2}\left\|\boldsymbol{\pi}_{i}^{\prime}-\boldsymbol{\alpha}_{i}^{\prime}\right\|_{2}^{2}\right] \\
& +\beta\left(\sum_{j \in \overline{\mathcal{M}}} t_{j}\right)+\frac{c}{2}\left(\sum_{j \in \overline{\mathcal{M}}} t_{j}\right)^{2} \\
& +\sum_{j \in \overline{\mathcal{M}}}\left[\psi_{j}\left(\mathbf{b}_{j}^{T} \boldsymbol{\pi}_{j}-\frac{1}{M+1}-t_{j}\right)\right. \\
& +\frac{c}{2}\left(\mathbf{b}^{T} \boldsymbol{\pi}_{j}-\frac{1}{M+1}-t_{j}\right)^{2} \\
& \left.+\boldsymbol{\delta}_{j}^{T}\left(\boldsymbol{\pi}_{j}-\boldsymbol{\alpha}_{j}\right)+\frac{c}{2}\left\|\boldsymbol{\pi}_{j}-\boldsymbol{\alpha}_{j}\right\|_{2}^{2}\right]
\end{aligned}
$$

where $c>0$ is the penalty parameter, $\left\{\psi_{j}\right\}_{j \in \overline{\mathcal{N}}},\left\{\zeta_{i}\right\}_{i \in \mathcal{N}}$, $\left\{\boldsymbol{\delta}_{j}\right\}_{j \in \overline{\mathcal{M}}}$, and $\beta$ are the Lagrangian multipliers. The ADMM is an iterative algorithm where local variables are updated (with others being fixed) in each iteration until convergence. In the following we present the updating procedure at the $m$ th iteration of the proposed ADMM. Specifically, variables $\left(\tilde{\boldsymbol{\Psi}}_{i}, \tilde{\boldsymbol{\Lambda}}_{i}, \boldsymbol{\pi}_{i}^{\prime}\right)$ are updated at user $i$ by solving the following convex subproblem

$$
\begin{aligned}
\underset{\left(\tilde{\boldsymbol{\Psi}}_{i}, \tilde{\boldsymbol{\Lambda}}_{i}, \boldsymbol{\pi}_{i}^{\prime}\right) \in \mathcal{O}_{i}}{\operatorname{minimize}} f_{i}\left(\hat{\phi}_{i}, \tilde{\boldsymbol{\Lambda}}_{i}\right) & +\left(\zeta_{i}^{(m-1)}\right)^{T} \boldsymbol{\pi}_{i}^{\prime} \\
& +\frac{c}{2}\left\|\boldsymbol{\pi}_{i}^{\prime}-\boldsymbol{\alpha}_{i}{ }^{\prime(m-1)}\right\|_{2}^{2} .
\end{aligned}
$$

And for updating variables $\pi_{j}$, BS $j$ solves the subproblem

$$
\begin{gathered}
\underset{\boldsymbol{\pi}_{j} \in \tilde{\mathcal{O}}_{j}}{\operatorname{minimize}} \psi_{j}^{(m-1)} \mathbf{b}_{j}^{T} \boldsymbol{\pi}_{j}+\frac{c}{2}\left(\mathbf{b}_{j}^{T} \boldsymbol{\pi}_{j}-\frac{1}{M+1}-t_{j}^{(m-1)}\right)^{2} \\
+\left(\boldsymbol{\delta}_{j}^{(m-1)}\right)^{T} \boldsymbol{\pi}_{j}+\frac{c}{2}\left\|\boldsymbol{\pi}_{j}-\boldsymbol{\alpha}_{j}^{(m-1)}\right\|_{2}^{2}
\end{gathered}
$$

which is a quadratic programming problem. We remark that the subproblems (24) and (25) can be solved independently at users and BSs, respectively. 
The following step is to update variables $\mathbf{t}$, for which we need to solve the problem

$$
\begin{aligned}
\mathbf{t}^{(m)}= & \underset{\mathbf{t}}{\arg \min } \sum_{j \in \overline{\mathcal{M}}}\left(\frac{c}{2}\left(\mathbf{b}_{j}^{T} \boldsymbol{\pi}_{j}^{(m)}-\frac{1}{M+1}-t_{j}\right)^{2}\right. \\
& \left.-\psi_{j}^{(m-1)} t_{j}+\beta^{(m-1)} t_{j}\right)+\frac{c}{2}\left(\sum_{j \in \overline{\mathcal{M}}} t_{j}\right)^{2} .
\end{aligned}
$$

Interestingly, (26) can be solved by closed-form expressions as follows [34]. Let $z_{j}^{(m)}=\mathbf{b}_{j}^{T} \pi_{j}^{(m)}-\frac{1}{M+1}+\frac{\psi_{j}^{(m-1)}-\beta^{(m-1)}}{c}$, then we can rewrite (26) as

$$
\mathbf{t}^{(m)}=\underset{\mathbf{t}}{\arg \min } \sum_{j \in \overline{\mathcal{M}}}\left(t_{j}-z_{j}^{(m)}\right)^{2}+\left(\sum_{j \in \overline{\mathcal{M}}} t_{j}\right)^{2}
$$

which is equivalent to the following problem

$$
\begin{array}{ll}
\underset{\mathbf{t}, \bar{t}}{\operatorname{minimize}} & \sum_{j \in \overline{\mathcal{M}}}\left(t_{j}-z_{j}^{(m)}\right)^{2}+((M+1) \bar{t})^{2} \\
\text { subject to } & \frac{1}{M+1} \sum_{j \in \overline{\mathcal{M}}} t_{j}=\bar{t} .
\end{array}
$$

Given $\bar{t}$, the optimal solution of this problem is given by

$$
t_{j}^{(m)}=\bar{t}+z_{j}^{(m)}-\bar{z}^{(m)}
$$

where $\bar{z}^{(m)}=\frac{\sum_{j \in \overline{\mathcal{M}}} z_{j}^{(m)}}{1+M}$. Consequently, we have the problem

$$
\underset{\bar{t}}{\operatorname{minimize}}\left(\bar{t}-\bar{z}^{(m)}\right)^{2}+(M+1) \bar{t}^{2}
$$

which has the solution $\bar{t}=\bar{z}^{(m)} /(M+2)$. Finally, $t_{j}^{(m)}$ is calculated as

$$
t_{j}^{(m)}=z_{j}^{(m)}-\bar{z}^{(m)}(M+1) /(M+2)
$$

where $\bar{z}^{(m)}$ can be achieved by running an average consensus algorithm [34], [35]. And thus, updating $t_{j}$ can be taken place at BS $j$.

To update the global variables $\left\{\boldsymbol{\alpha}_{j}\right\}$ (recall that $\left\{\boldsymbol{\alpha}_{j}\right\}$ and $\left\{\boldsymbol{\alpha}_{i}^{\prime}\right\}$ are derived from the same set of variables, i.e. $\phi$ and $\left.\left\{s_{i j k}\right\}\right)$, the following problem is solved

$$
\begin{aligned}
\left\{\boldsymbol{\alpha}_{j}^{(m)}\right\}= & \underset{\left\{\boldsymbol{\alpha}_{j}\right\}}{\arg \min } \sum_{i \in \mathcal{N}}\left(-\left(\zeta_{i}^{(m-1)}\right)^{T} \boldsymbol{\alpha}_{i}^{\prime}+\frac{c}{2}\left\|\boldsymbol{\pi}_{i}{ }^{(m)}-\boldsymbol{\alpha}_{i}^{\prime}\right\|_{2}^{2}\right) \\
& +\sum_{j \in \mathcal{M}}\left(-\left(\boldsymbol{\delta}_{j}^{(m-1)}\right)^{T} \boldsymbol{\alpha}_{j}+\frac{c}{2}\left\|\boldsymbol{\pi}_{j}^{(m)}-\boldsymbol{\alpha}_{j}\right\|_{2}^{2}\right) .
\end{aligned}
$$

This is in fact an unconstrained quadratic programming problem, for which the solution is given by

$$
\begin{aligned}
\phi^{(m)} & =\frac{\sum_{i \in \mathcal{N}} u_{i}+\sum_{j \in \overline{\mathcal{M}}} \tilde{u}_{j}}{c(N+M+1)}, \\
s_{i j k}^{(m)} & =\frac{\left[\zeta_{i}^{(m-1)}\right]_{v_{i j k}^{\prime}}+\left[\delta_{j}^{(m-1)}\right]_{v_{i j k}}}{2 c}+\frac{v_{i j k}{ }^{\prime(m)}+v_{i j k}^{(m)}}{2}
\end{aligned}
$$

Algorithm 2. The proposed distributed algorithm based ADMM

1: Initialization: Set $m=0$ and choose initial values for $\left\{\boldsymbol{\alpha}_{j}^{(0)}\right\},\left\{\psi_{j}^{(0)}\right\},\left\{\zeta_{i}^{(0)}\right\},\left\{\delta_{j}^{(0)}\right\}, \beta^{(0)}$.

\section{2: repeat}

3: $\quad m:=m+1$.

4: $\quad$ User $i$ updates $\left(\tilde{\boldsymbol{\Psi}}_{i}, \tilde{\boldsymbol{\Lambda}}_{i}, \boldsymbol{\pi}_{i}^{\prime}\right)$ as in (24); BS $j$ updates $\boldsymbol{\pi}_{j}$ as in (25).

5: $\quad$ BS $j$ computes $z_{j}$, determines $\bar{z}$ by running an average consensus algorithm among BSs, and then updates $t_{j}$ by (31).

6: BSs update $\phi$ by (33) through an average consensus algorithm.

7: $\quad$ BS $j$ receives $\left[\zeta_{i}^{(m-1)}\right]_{v_{i j k}^{\prime}}+c v_{i j k^{(m)}}$ from user $i \in \mathcal{N}_{j}$, and then updates $s_{i j k}$ by (34).

8: User $i$ receives $\left\{s_{i j k}\right\}_{j, k}$ from BSs to form $\boldsymbol{\alpha}_{i}{ }^{\prime(m)}$, then updates $\zeta_{i}$ by (35)

9: $\quad$ BS $j$ update $\psi_{j}, \boldsymbol{\delta}_{j}, \beta$ use (36), (37), and (38), respectively.

10: until Convergence.

where $u_{i}=\left(\left[\zeta_{i}^{(m-1)}\right]_{\hat{\phi}_{i}}+c \hat{\phi}_{i}^{(m)}\right), \tilde{u}_{j}=\left(\left[\delta_{j}^{(m-1)}\right]_{\tilde{\phi}_{j}}+c \tilde{\phi}_{j}^{(m)}\right)$, $\left[\zeta_{i}^{(m-1)}\right]_{\hat{\phi}_{i}}$ is the element of $\zeta_{i}^{(m-1)}$ corresponding to constraint $\phi=\hat{\phi}_{i}$, and similar definitions apply to $\left[\delta_{j}^{(m-1)}\right]_{\tilde{\phi}_{j}}$, $\left[\zeta_{i}^{(m-1)}\right]_{v_{i j k}^{\prime}}$, and $\left[\delta_{j}^{(m-1)}\right]_{v_{i j k}}$. The value of $\phi$ can also be updated by performing an average consensus algorithm among all BSs and users. For calculating variable $s_{i j k}$, BS $j$ can gather the value $\left[\zeta_{i}^{(m-1)}\right]_{v_{i j k}^{\prime}}+c v_{i j k}{ }^{\prime(m)}$ from all users $i \in \mathcal{N}_{j}$.

The last step of the ADMM is to update the Lagrangian multipliers, which is simply found as

$$
\begin{aligned}
\zeta_{i}^{(m)} & =\zeta_{i}^{(m-1)}+c\left(\boldsymbol{\pi}_{i}{ }^{\prime(m)}-\boldsymbol{\alpha}_{i}{ }^{(m)}\right), \\
\psi_{j}^{(m)} & =\psi_{j}^{(m-1)}+c\left(\mathbf{b}^{T} \boldsymbol{\pi}_{j}^{(m)}-\frac{1}{M+1}-t_{j}^{(m)}\right), \\
\boldsymbol{\delta}_{j}^{(m)} & =\boldsymbol{\delta}_{j}^{(m-1)}+c\left(\boldsymbol{\pi}_{j}^{(m)}-\boldsymbol{\alpha}_{j}^{(m)}\right), \\
\beta^{(m)} & =\beta^{(m-1)}+c \frac{M+1}{M+2} \bar{z}^{(m)} .
\end{aligned}
$$

We remark that $\zeta_{i}$ is updated at user $i$ while $\left(\psi_{j}, \boldsymbol{\delta}_{j}, \beta\right)$ are updated at $\mathrm{BS} j$. In particular, $\beta$ can be updated at each BS since the value of $\bar{z}^{(m)}$ is available at all BSs when $\left\{t_{j}\right\}$ is updated as mentioned previously. The main steps of the proposed distributed method solving (19) are outlined in Algorithm 2.

We summarize the information exchanged between nodes for running Algorithm 2 in Table I. As can be observed, the amount of exchanged information in the network is mainly due to the two average consensus algorithms for updating variables $\left\{t_{j}\right\}$ and $\phi$ corresponding to steps 5 and 6 , respectively. In particular, the overhead for exchanging relevant information in each step of the first and second consensus algorithms is $M+1$ and $M+N+1$, respectively [35]. On the other hand, the complexity of the algorithm comes from step 4, i.e. to solve convex 
TABLE. I

INFORMATION EXCHANGE IN ALGORITHM 2

\begin{tabular}{c|c}
\hline Procedure & Exchanged information \\
\hline \hline Initial stage, derive $\mathbf{B}_{i}$ at user $i$ & $\left\{\overline{\mathbf{H}}_{i j}\right\}$, from BSs in $\overline{\mathcal{M}}_{j}$ to user $i$ \\
\hline Step 5, update $t_{j}$ at BS $j$ & $\left\{z_{j}\right\}$ (average consensus between BSs) \\
\hline Step 6, update $\phi$ & $\left\{u_{i}\right\},\left\{\tilde{u}_{j}\right\}$ (average consensus between BSs and users) \\
\hline Step 7, update $s_{i j k}$ & {$\left[\zeta_{i}^{(m-1)}\right]_{v_{i j k}^{\prime}}+c v_{i j k}^{(m)}$, from user $i$ to BS $j$} \\
\hline Step 8, update $\zeta_{i}$ & $\left\{s_{i j k}\right\}_{j, k}$, from BSs in $\overline{\mathcal{M}}_{j}$ to user $i$ to form $\boldsymbol{\alpha}_{i}^{\prime}$ \\
\hline
\end{tabular}

subproblems (24) and (25) at users and BSs, respectively. Since (24) is a generic convex program this problem can be solved with the worst complexity of $\mathcal{O}\left(\left(\sum_{j \in \overline{\mathcal{M}}_{i}} K_{j}+L_{i}^{2}+\hat{L}_{i}^{2}\right)^{4}\right)$ [31, Sect. 6]. Similarly, (25) is a quadratic problem which can be reformulated as a second-order cone program, and thus the cost of solving this problem is $\mathcal{O}\left(\left|\mathcal{N}_{j}\right|^{3} K_{j}^{3}\right)$ [36].

We now present briefly the convergence of Algorithm 2. Recall that problem (7) is supposed to be strictly feasible (by a proper scheduler), and so is problem (18) since these two problems are equivalent (i.e. strong duality holds for (18)). Consequently, the unaugmented Lagrangian of (19) (obtained from (23) by setting $c=0$ ) has a saddle point. In addition, we note that (24) and (25) are solvable. Thus, the convergence proof of Algorithm 2 to the optimal solution of (19) is straightforward, following the same arguments as in [20, Appendix A].

\section{JOINT PRECOder Design AND BS SELECTION}

We have considered the EEmax problem with a fully connected network, where all BSs are switched on and transmit data to all their serving users. In small-cell networks, a user can receive information from both macro-cell BS and smallcell BSs. This means, information is still transmitted to the user when some small-cell BSs are turned off (but the data rate can be reduced). In addition, when accounting for the circuit power, shutting down some small-cell BSs whose channels suffer deep fading could improve the energy efficiency performance. This is because the added data transmission capacity brought by these BSs is not commensurate with the circuit power consumption required to keep these $\mathrm{BSs}$ in active mode. These discussions motivate us to investigate the joint precoder design and BS selection (JPBS) problem to enhance the network energy efficiency in this section.

To formulate the JPBS problem, we adopt a new power consumption model that includes the power required to turn off and on BSs [37]. Let $\rho_{j} \in\{0,1\}$ indicate BS $j$ is turned on and off when $\rho_{j}=1$ and $\rho_{j}=0$, respectively. We also introduce the binary variable $\varphi_{i j} \in\{0,1\}$ to indicate $\mathrm{BS} j$ is assigned to transmit data to user $i$ with $\varphi_{i j}=1$, and $\varphi_{i j}=0$ otherwise. Let $p_{j}^{\text {cirSw }}$ be the power required to switch off and on BS $j$. Then, the total circuit power is given by [37]

$$
\begin{aligned}
P^{\mathrm{cir}}=\sum_{j \in \overline{\mathcal{M}}}( & \left.\left(1-\rho_{j}\right) p_{j}^{\mathrm{cirSw}}+\rho_{j}\left(p_{j}^{\mathrm{cirTx}}+\sum_{i \in \mathcal{N}_{j}} \varphi_{i j} p_{i j}^{\mathrm{cirCo}}\right)\right) \\
& +\sum_{i \in \mathcal{N}} p_{i}^{\mathrm{cirRx}} .
\end{aligned}
$$

We are interested in the practical case of $p_{j}^{\text {cirSw }}<p_{j}^{\text {cirTx }}$, meaning that turning off $\mathrm{BS} j$ with $p_{j}^{\text {cirSw }}<p_{j}^{\text {cirTx }}$ is power efficient. In the JPBS problem we need to make sure that if $\mathrm{BS} j$ is turned off, it does not serve any user, i.e., if $\rho_{j}=0$ then $\varphi_{i j}=0$, for all $i \in \mathcal{N}_{j}$. Similarly, if BS $j$ does not send data to user $i$, then no power will be allocated to the link between them, i.e., if $\varphi_{i j}=0$ then $\operatorname{Tr}\left(\tilde{\mathbf{B}}_{i j} \boldsymbol{\Psi}_{i} \tilde{\mathbf{B}}_{i, j}^{H}\right)=0$. From the above discussions, we now can state the JPBS problem as

$$
\begin{aligned}
& \underset{\left\{\mathbf{G}_{i}\right\},\left\{\rho_{j}\right\},\left\{\varphi_{i j}\right\}}{\operatorname{maximize}} \frac{\sum_{i \in \mathcal{N}} a_{i} \log \left|\mathbf{I}+\tilde{\mathbf{H}}_{i} \boldsymbol{\Psi}_{i} \tilde{\mathbf{H}}_{i}^{H}\right|}{\sum_{j \in \overline{\mathcal{M}}} \frac{1}{\lambda_{j}} \sum_{i \in \mathcal{N}} \operatorname{Tr}\left(\tilde{\mathbf{B}}_{i j} \boldsymbol{\Psi}_{i} \tilde{\mathbf{B}}_{i j}^{H}\right)+P^{\mathrm{cir}}} \\
& \text { subject to } \log \left|\mathbf{I}+\tilde{\mathbf{H}}_{i} \boldsymbol{\Psi}_{i} \tilde{\mathbf{H}}_{i}^{H}\right| \geq \bar{R}_{i}, \forall i, \\
& \sum_{i \in \mathcal{N}_{j}} \operatorname{Tr}\left(\tilde{\mathbf{B}}_{i j} \boldsymbol{\Psi}_{i} \tilde{\mathbf{B}}_{i, j}^{H}\right) \leq \rho_{j} P_{j}, \forall j, \\
& \operatorname{Tr}\left(\tilde{\mathbf{B}}_{i j} \boldsymbol{\Psi}_{i} \tilde{\mathbf{B}}_{i, j}^{H}\right) \leq \varphi_{i j} P_{j}, \forall i, j, \\
& \sum_{i \in \mathcal{N}_{j}}\left[\tilde{\mathbf{B}}_{i j} \boldsymbol{\Psi}_{i} \tilde{\mathbf{B}}_{i j}^{H}\right]_{k, k} \leq \rho_{j} P_{j k}, \forall k, j, \\
& \varphi_{i j} \leq \rho_{j}, \forall i, j ; \sum_{j \in \overline{\mathcal{M}}_{i}} \varphi_{i j} \geq 1, \\
& \varphi_{i j} \in\{0,1\}, \rho_{j} \in\{0,1\}, \forall i, j .
\end{aligned}
$$

We note that zero-forcing technique is still applied in this section, and the matrices $\left\{\tilde{\mathbf{B}}_{i j}\right\}$ are calculated based on the set of active BSs, i.e. $\left\{\tilde{\mathbf{B}}_{i j}\right\}$ must be redetermined when there is a BS changes its state. In order words, $\left\{\tilde{\mathbf{B}}_{i j}\right\}$ are functions of $\left\{\rho_{j}\right\}$. Problem (40) is a mixed Boolean nonlinear program [22], and thus to find an optimal solution is challenging. In the next subsection, we present a tailored BnB method to solve the JPBS problem optimally.

\section{A. Optimal Algorithm via Branch-and-Bound Method}

We made two important observations regarding the JPBS problem in (40). First, if $\rho_{j}$ 's and $\varphi_{i j}$ 's are fixed to 0 or 1 , then the JPBS problem is an instant of the EEmax problem with a fixed configuration which can be solved efficiently using the methods presented in the preceding section. Second, due to (40f), we can equivalently rewrite the circuit power in (39) as

$$
\begin{aligned}
P^{\mathrm{cir}} & =\sum_{j \in \overline{\mathcal{M}}} p_{j}^{\mathrm{cirSw}}+\sum_{i \in \mathcal{N}} p_{i}^{\mathrm{cirRx}} \\
& +\sum_{j \in \overline{\mathcal{M}}}\left(\rho_{j}\left(p_{j}^{\mathrm{cirTx}}-p_{j}^{\mathrm{cirSw}}\right)+\sum_{i \in \mathcal{N}_{j}} \varphi_{i j} p_{i j}^{\mathrm{cirCo}}\right)
\end{aligned}
$$




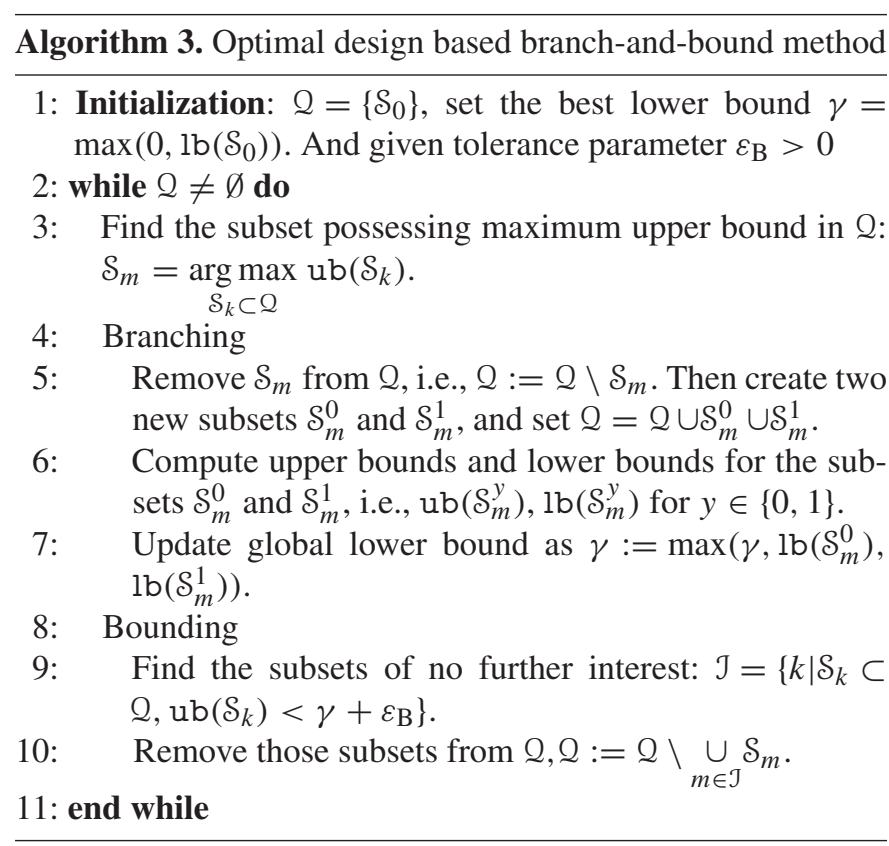

which is a linear function of $\rho_{j}$ and $\varphi_{i j}$. Thus, if $\rho_{j}$ and $\varphi_{i j}$ are relaxed to be continuous on the interval $[0,1]$, then problem (40) becomes a concave-convex fractional program, which can also be solved by slightly modifying the proposed methods introduced in the previous section. The two remarks are used to find upper and lower bounds of the JPBS problem in each step of the $\mathrm{BnB}$ method.

In general, the $\mathrm{BnB}$ method obtains an optimal solution by dividing the feasible set into subsets, calculating upper and lower bounds of these subsets (branching step), and removing those that do not contain an optimal solution (bounding step). We outline the proposed BnB method solving (40) in Algorithm 3, and refer the interested reader to [21], [22] for further details on the general framework of $\mathrm{BnB}$ methods. Some notations have been introduced in Algorithm 3. Specifically, for a subset $\mathcal{S}, \mathrm{ub}(\mathcal{S})$ and $\mathrm{lb}(\mathcal{S})$ denote upper bound and lower bound of the JPBS problem over $\mathcal{S}$, respectively. $\gamma$ is the largest lower bound of all subsets which have been considered, and $Q$ contains the subsets whose upper bounds are larger than $\gamma$. At the initial stage, $\mathcal{Q}$ contains only $\mathcal{S}_{0}$ which is the feasible set of (40) and $\gamma$ is set to

$$
\gamma=\max \left(0, \mathrm{lb}\left(\mathcal{S}_{0}\right)\right) .
$$

The main steps of Algorithm 3 are detailed in the following.

Branching step: In this step, a subset in $Q$ is divided into two new subsets, and then upper and lower bounds of these new subsets are determined. Suppose subset $\mathcal{S}_{m}$ has the largest upper bound among all subsets in $\mathcal{Q}$ (line 3 ). Let $\mathcal{U}\left(\mathcal{S}_{m}\right)$ be the set of unfixed Boolean variables (unfixed $\rho_{j}$ and $\varphi_{i j}$ ) of $\mathcal{S}_{m}$, then $\mathcal{S}_{m}$ is divided into two subsets $\mathcal{S}_{m}^{1}$ and $\mathcal{S}_{m}^{0}$ as

$$
\mathcal{S}_{m}^{y}=\left\{\mathcal{S}_{m} \mid w_{t}=y, w_{t} \in \mathcal{U}\left(\mathcal{S}_{m}\right)\right\}, \text { for } y \in\{0,1\}
$$

where $w_{t}$ is an arbitrary unfixed binary variable in $\mathcal{U}\left(\mathcal{S}_{m}\right)$. The convergence of the $\mathrm{BnB}$ method is guaranteed even when $w_{t}$ is randomly chosen. However, an appropriate branching rule that is developed for a specific problem may remarkably accelerate the convergence. In the spirit of [34] we define the virtual energy efficiency for BS $j$ as

$$
\mathcal{E}_{\mathrm{BS}}\left(\rho_{j}\right)=\frac{\sum_{i \in \mathcal{N}_{j}} \log \left|\mathbf{I}+\check{\mathbf{H}}_{i j} \boldsymbol{\Psi}_{i} \check{\mathbf{H}}_{i j}^{H}\right|}{\frac{1}{\lambda_{j}} \sum_{i \in \mathcal{N}_{j}} \operatorname{Tr}\left(\tilde{\mathbf{B}}_{i j} \boldsymbol{\Psi}_{i} \tilde{\mathbf{B}}_{i, j}^{H}\right)+p_{j}^{\text {cirTx }}-p_{j}^{\text {cirSw }}} .
$$

where $\check{\mathbf{H}}_{i j}=\frac{1}{\sigma_{i}} \mathbf{H}_{i j} \tilde{\mathbf{B}}_{i j}$. Similarly, for variable $\varphi_{i j}$, the virtual link energy efficiency is defined as

$$
\varepsilon_{\text {link }}\left(\varphi_{i j}\right)=\frac{\log \left|\mathbf{I}+\check{\mathbf{H}}_{i j} \boldsymbol{\Psi}_{i} \check{\mathbf{H}}_{i j}^{H}\right|}{\frac{1}{\lambda_{j}} \operatorname{Tr}\left(\tilde{\mathbf{B}}_{i j} \boldsymbol{\Psi}_{i} \tilde{\mathbf{B}}_{i, j}^{H}\right)+p_{i j}^{\text {cirCo }}} .
$$

Then, we propose the following branching rule

$$
w_{t}=\underset{w \in \mathcal{U}\left(\mathcal{S}_{m}^{y}\right)}{\arg \min }\left\{\mathcal{E}_{\mathrm{BS}}(w) \text { and } \mathcal{E}_{\text {link }}(w)\right\}
$$

which means that the subset that has smallest energy efficiency contribution is to be branched. The lower and upper bounds of subset $\mathcal{S}_{m}^{y}, y \in\{0,1\}$, are computed as follows.

Upper bound: An upper bound of the JPBS problem over subset $\mathcal{S}_{m}^{y}$ can be obtained by simply relaxing Boolean variables in $\mathcal{U}\left(\mathcal{S}_{m}^{y}\right)$, i.e. $0 \leq \rho_{j} \leq 1,0 \leq \varphi_{i j} \leq 1, \forall \rho_{j}, \varphi_{i j} \in \mathcal{U}\left(\mathcal{S}_{m}^{y}\right)$. We recall that the resulting problem can be solved optimally as mentioned above. If the relaxed problem for $U\left(\mathcal{S}_{m}^{y}\right)$ is infeasible, we set ub $\left(\mathcal{S}_{m}^{y}\right)<0$, thus $\mathcal{S}_{m}^{y}$ will be removed from $\mathcal{Q}$ at bounding step.

Lower bound: Calculating a lower bound of JPBS problem corresponding to $\mathcal{S}_{m}^{y}$ can be done by several ways. In this paper a lower bound is found by simply rounding the nonbinary solutions, which are obtained after solving the relaxed problem, to 0 and 1 [22]. If $\mathrm{lb}\left(\mathcal{S}_{m}^{y}\right)>\gamma$, we set $\gamma=\mathrm{lb}\left(\mathcal{S}_{m}^{y}\right)$.

Bounding step: After updating $\gamma$, the subsets, whose upper bounds are smaller than $\gamma$, are removed from $\mathcal{Q}$ (line 9). The iterative procedure continues until $Q=\emptyset$.

\section{B. Low-Complexity Suboptimal Design}

Although the proposed $\mathrm{BnB}$ algorithm presented in the previous subsection remarkably reduces the computational complexity compared to an exhaustive search method, its complexity is still too high for practical implementation, especially for moderate and large-scale networks. This motivates us to design a low-complexity suboptimal algorithm which can attain a near-optimal performance in polynomial time.

The proposed low-complexity algorithm is based on the idea of greedy selection. It can be observed from (40) that if one BS does not serve any user, it should be turned off, i.e. if $\varphi_{i j}=0, \forall i \in \mathcal{N}_{j}$ then $\rho_{j}=0$. On the other hand, if $\varphi_{i j}=1$ for some $i \in \mathcal{N}_{j}$ then $\rho_{j}=1$. From this observation as well as the definition of BS energy efficiency and link energy efficiency in (44) and (45), respectively, we propose a greedy algorithm to find a solution to the JPBS problem as follows. Let $\tilde{\mathcal{J}}_{\mathrm{BS}}$ and $\tilde{\mathcal{J}}_{\mathrm{Co}}$ denote the sets of current active BSs and unconsidered links, respectively. At the initial stage, we consider a fully connected network where all BSs are switched on and 


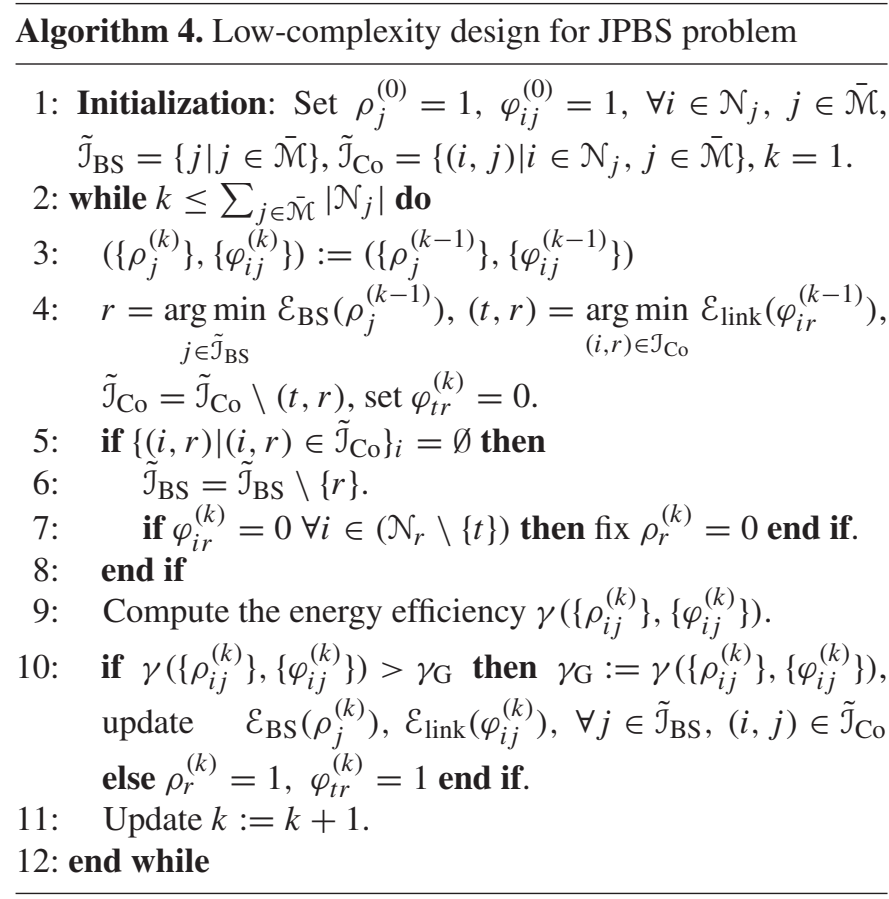

all transmission links are available, i.e., $\rho_{j}=1, \varphi_{i j}=1, \forall i \in$ $\mathcal{N}_{j}, j \in \overline{\mathcal{M}}, \tilde{\mathcal{J}}_{\mathrm{BS}}=\{j \mid j \in \overline{\mathcal{M}}\}, \tilde{\mathcal{J}}_{\mathrm{Co}}=\left\{(i, j) \mid i \in \mathcal{N}_{j}, j \in \overline{\mathcal{M}}\right\}$. Let $\gamma_{\mathrm{G}}$ denote the current best energy efficiency. At the initial stage, $\gamma_{\mathrm{G}}$ is the solution of the problem corresponding to the fully-connected network. In the next iterations, we gradually turn off one coordination link if doing so is energy-efficient. Specifically, let $\gamma\left(\left\{\rho_{j}^{(k)}\right\},\left\{\varphi_{i j}^{(k)}\right\}\right)$ denote the energy efficiency corresponding to $\left(\left\{\rho_{j}^{(k)}\right\},\left\{\varphi_{i j}^{(k)}\right\}\right)$ at the $k$ th iteration. Herein, we set $\gamma\left(\left\{\rho_{j}^{(k)}\right\},\left\{\varphi_{i j}^{(k)}\right\}\right)<0$ if the resulting problem is infeasible. If $\gamma\left(\left\{\rho_{j}^{(k)}\right\},\left\{\varphi_{i j}^{(k)}\right\}\right)>\gamma_{\mathrm{G}}$, then the considered coordination link is switched off. We update $\gamma_{\mathrm{G}}$ and continue the iterative procedure. The proposed greedy algorithm is described in Algorithm 4. To find the considered link in an iteration, we first determine the BS who possesses the least energy efficiency contribution, then the coordination link corresponding to this BS and contributing the least energy efficiency is chosen (line 4). Line 7 indicates the case where a BS is turned off since it is found not to serve any user.

It is obvious that Algorithm 4 stops when all possible links have been considered, i.e. after $\sum_{j \in \overline{\mathcal{M}}}\left|\mathcal{N}_{j}\right|$ iterations. We note that the complexity in each iteration depends mainly on computing the energy efficiency $\gamma\left(\left\{\rho_{i j}^{(k)}\right\},\left\{\varphi_{i j}^{(k)}\right\}\right)$, which is obtained by solving a EEmax problem corresponding to $\left(\left\{\rho_{j}^{(k)}\right\},\left\{\varphi_{i j}^{(k)}\right\}\right)$. As shown in Section III this problem can be reformulated as a convex program which can be efficiently solved by an interiorpoint method [26]. In particular, the worst cost for computing $\gamma\left(\left\{\rho_{i j}^{(k)}\right\},\left\{\varphi_{i j}^{(k)}\right\}\right)$ (by solving (18) is $\mathcal{O}\left(\left(\sum_{i \in \mathcal{N}}\left(L_{i}^{2}+\hat{L}_{i}^{2}\right)\right)^{4}\right)$ [31, Sect. 6]. That is to say Algorithm 4 can solve the JPBS problem in polynomial time.

We also remark that Algorithm 4 can be carried out in a distributed manner as follows. First, the problem corresponding to $\gamma\left(\left\{\rho_{i j}^{(k)}\right\},\left\{\varphi_{i j}^{(k)}\right\}\right)$ (line 9) can be solved distributively by
Algorithm 2 presented in Section III-B. If $\gamma\left(\left\{\rho_{i j}^{(k)}\right\},\left\{\varphi_{i j}^{(k)}\right\}\right)>$ $\gamma_{\mathrm{G}}$ then users send the obtained precoding to the corresponding BSs. Next, each BS $j$ in the set of surviving BSs can easily obtain $\mathcal{E}_{\mathrm{BS}}\left(\rho_{j}^{(k)}\right)$ and $\mathcal{E}_{\text {link }}\left(\varphi_{i j}^{(k)}\right)$ based on (44) and (45), respectively. To determine BS $r$ as written in line 4 , it requires exchanging a scalar between active BSs by, e.g., backhaul links [1], [2]. After that, BS $r$ can determine the next considered link $(t, r)$ by itself.

\section{Performance Evaluation}

In this section we numerically evaluate the performance of the proposed algorithms. For comparison purpose, we consider the problem of power minimization (SPmin) and weight sum rate maximization (SRmax), which are given by $\operatorname{SPmin}=\min \left\{\sum_{j} \frac{1}{\lambda_{j}} \sum_{i} \operatorname{Tr}\left(\tilde{\mathbf{B}}_{i j} \boldsymbol{\Psi}_{i} \tilde{\mathbf{B}}_{i j}^{H}\right) \mid(7 \mathrm{~b})-\right.$ (7d) $\} \quad$ and $\quad \operatorname{SRmax}=\max \left\{\sum_{i} a_{i} \log \left|\mathbf{I}+\hat{\mathbf{H}}_{i} \boldsymbol{\Psi}_{i} \hat{\mathbf{H}}_{i}^{H}\right| \mid(7 \mathrm{~b})-\right.$ (7d)\}, respectively. We note that SPmin and SRmax are the approaches presented in [6] and [38], respectively, which are modified corresponding to the considered system for a fair comparison. In particular, the scheme in [6] is extended to MIMO case and the power constraints also include PAPCs. Similarly, QoS constraints of users and PAPCs are added to the scheme in [38]. The SPmin and SRmax problems are convex and thus can be solved in a similar manner to the EEmax problem. Moreover, we also compare the proposed methods with the one introduced in [14], which is basically a power allocation scheme based on fixed ZF beamformers. We refer to this scheme as ZF-PA in the rest of the paper.

The simulation parameters are provided in Table II, which mainly follow those studied in prior works. In particular, the propagation parameters are from [1, Table 2-1], [5], and the circuit powers are from [5], [10], [39]. ${ }^{3}$ For ease of description, we stack the QoS thresholds in a vector $\overline{\mathbf{r}}=\left[\bar{R}_{1}, \bar{R}_{2}, \ldots, \bar{R}_{N}\right]^{T}$. Other specific parameters are provided at the captions of figures.

In Fig. 2, we plot the duality gap of the interior-point and subgradient methods for solving convex subproblems (8) of the first iteration of Dinkelbach's method over two random channels. The duality gap of the interior-point method is attained by the SDPT3 solver [32]. As expected, the interior-point convex solver SDPT3 outperforms the subgradient method in terms of convergence rate. The subgradient method decreases the duality gap quickly for some first iterations, but the decrease becomes slow after that. This is due to the vibration effect of subgradient methods in the vicinity of an optimal solution. We remark that the subgradient method is merely based on closed-form expressions which require low computational complexity per iteration. Thus, the proposed subgradient method is suitable for the cases where moderate accuracy is acceptable.

Fig. 3 shows the convergence behavior of Algorithm 2 (ADMM) under two random channels with different values of

\footnotetext{
${ }^{3}$ Note that, we have omitted the bandwidth of the system in preceding sections to simplify the notations since it is a constant and thus has no impact on the proposed methods. LOS and NLOS in the table represent the terms line-ofsight and non-line-of-sight, respectively. $d$ is the distance between two nodes in $\mathrm{Km}$.
} 
TABLE. II

SimUlation PARAMETERS

\begin{tabular}{|c|c|}
\hline Parameter & Value \\
\hline System bandwidth & $10 \mathrm{MHz}$ \\
\hline Noise power density & $-174 \mathrm{dBm} / \mathrm{Hz}$ \\
\hline Radius of the macro-cell and the small-cells & $500 \mathrm{~m}$ and $40 \mathrm{~m}$ \\
\hline Distance between the macro BS and a small-cell BS & $>75 \mathrm{~m}$ \\
\hline Path loss from the macro BS to a user & $\begin{array}{ll}123.4+24.2 \log _{10}(d) & \text { for LOS } \\
151.1+42.8 \log _{10}(d) & \text { for NLOS }\end{array}(\mathrm{dB})$ \\
\hline Path loss from a small-cell BS to a user & $\begin{array}{ll}103.8+20.9 \log _{10}(d) & \text { for LOS } \\
145.4+37.5 \log _{10}(d) & \text { for NLOS }\end{array}(\mathrm{dB})$ \\
\hline Log normal shadowing standard deviation & 3 for LOS and 4 for NLOS \\
\hline The probability of LOS from the macro BS to a user & $\min \left(\frac{0.018}{d}, 1\right)\left(1-\exp \left(\frac{-d}{0.063}\right)\right)+\exp \left(\frac{-d}{0.063}\right)$ \\
\hline The probability of LOS from a small-cell BS to a user & $0.5-\min \left(0.5,5 \exp \left(-\frac{0.156}{d}\right)\right)+\min \left(0.5,5 \exp \left(-\frac{d}{0.03}\right)\right)$ \\
\hline Maximum transmit power of BSs and antennas & $P_{0}=46 \mathrm{dBm}, P_{j}=30 \mathrm{dBm}, P_{j, k}=P_{j} / K_{j}$ \\
\hline The efficiency of power amplifiers & $\lambda_{0}=39 \%, \lambda_{j}=7 \%$ \\
\hline The circuit power of idle mode & $p_{0}^{\operatorname{cirTx}}=24 \mathrm{dBm}, p_{j}^{\mathrm{cirTx}}=22.6 \mathrm{dBm}$ \\
\hline The receive circuit power of users & $p_{i}^{\mathrm{cirRx}}=20.4 \mathrm{dBm}$ \\
\hline The circuit power turning on and off BSs & $p_{j}^{\mathrm{cirSw}}=p^{\mathrm{cirSw}}=5 \mathrm{dBm}$ \\
\hline The circuit power of coordination & $p_{i j}^{\mathrm{cirCo}}=p^{\mathrm{cirCo}}, \forall i, j$ \\
\hline The priorities of users & $a_{i}=1, \forall i$, all users have the same priority \\
\hline
\end{tabular}

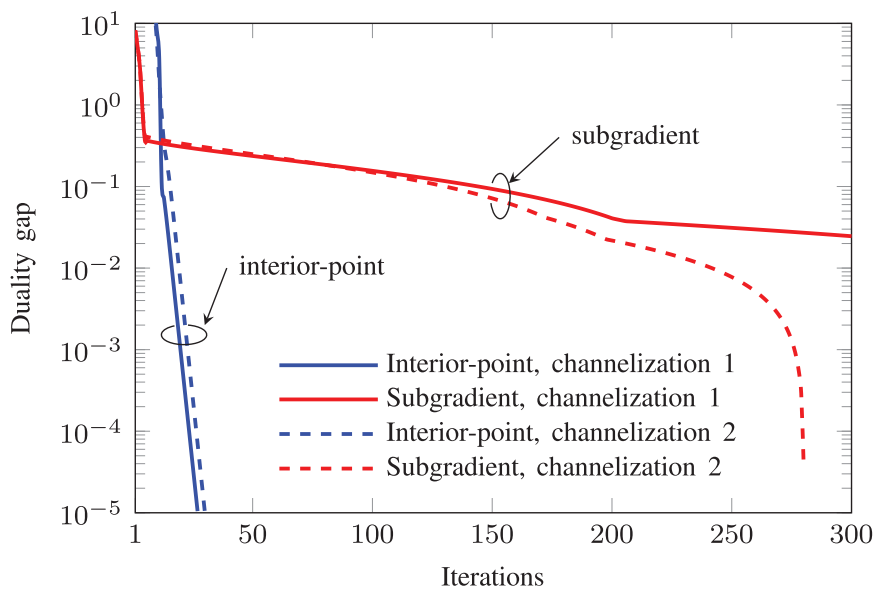

Fig. 2. Duality gap of interior-point and subgradient methods over two random channels. The network setting is $M=2, N=3, K_{0}=6, K_{1}=K_{2}=4$ and $L_{i}=2$ for all $i, \mathcal{N}_{1}=\{2\}, \mathcal{N}_{2}=\{3\}$, and user 1 is only served by the macro BS. The vector of QoS constraints of the users is $\overline{\mathbf{r}}=[14.43,28.85,15.87]$ Mbits/s, and $p^{\text {cirCo }}=5 \mathrm{dBm}$.

penalty parameter $c$. We can see in Fig. 3 that in all cases (channels and penalty parameters), the algorithm requires less than 30 iterations to converge.

In Fig. 4 we investigate the performances of EEmax, SRmax, SPmin, and ZF-PA schemes versus different coordinated circuit power, $p^{\text {cirCo }}$. The results in Fig. 4(a) clearly show the proposed EEmax scheme outperforms the other scheme of comparison in terms of energy efficiency. An interesting observation is that SPmin yields better energy efficiency than SRmax when $p^{\text {cirCo }}$ is small, and vice versa when $p^{\text {cirCo }}$ is large. We can explain this fact as follows. A small change in data dependent power would have a large impact on the denominator of the objective in (7) if the circuit power is small. However, data rate is a logarithmic function of power. Thus, an energy-efficient strategy in the low circuit power regime is to use a small amount of transmit power

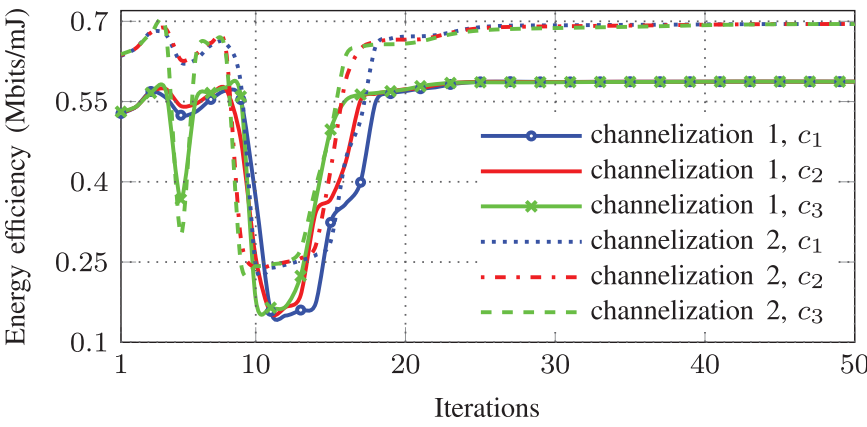

Fig. 3. Convergence results of Algorithm 2 (ADMM). The parameters are taken from Fig. 2. One iteration in the figure consists of all the procedure from step 3 to step 9 of the algorithm.

(as long as the QoS constraints are satisfied). On the contrary, the circuit power contributes mostly to the total consumption power when it is large, compared to the data dependent power. In this situation we can approximate the denominator as a constant. Therefore, for enhancing energy efficiency, the EEmax solution should use more transmit power to increase the numerator (i.e., sum rate). This explains the increased sum rate of the EEmax scheme with respect to circuit power in Fig. 4(b). Since the sum rates of SPmin and SRmax are independent of circuit powers as seen in Fig. 4(b), the energy efficiency of these schemes is reduced as $p^{\text {cirCo }}$ increases.

In Fig. 5 we investigate the performances of EEmax, SRmax, SPmin, and ZF-PA schemes versus QoS of data rate of users. Again, EEmax always outperforms the others in terms of energy efficiency, but its performance gains are reduced when the data rate thresholds $\bar{R}$ are set higher. The reason for this is that a higher amount of transmit power must be used to satisfy higher $\bar{R}$, but it provides a slight increase in the achieved sum rate. In case of SRmax, the considered values of $\bar{R}$ are not large enough 


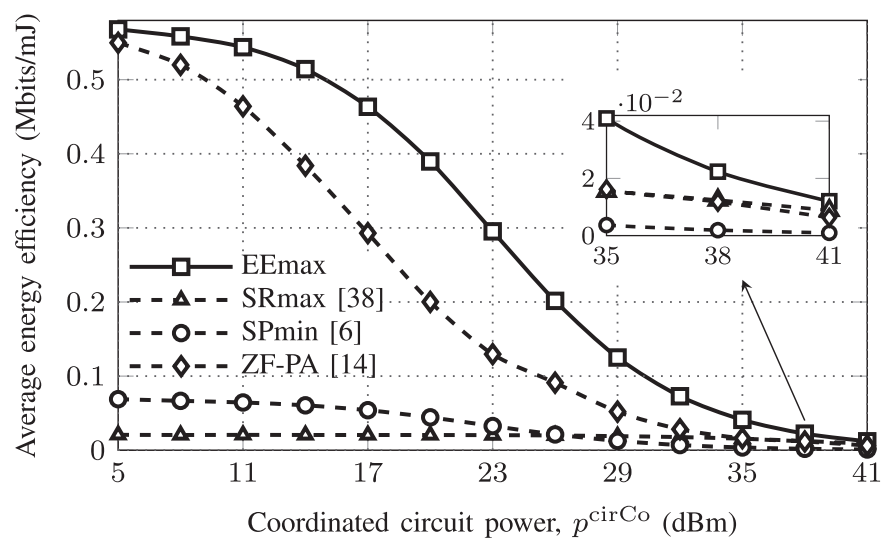

(a) Average energy-efficient performances

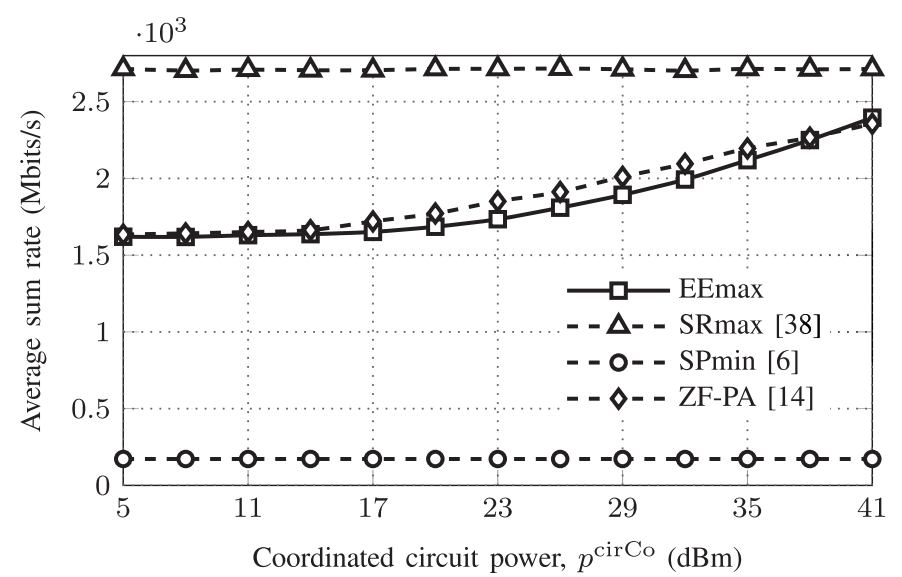

(b) Average sum rate performances

Fig. 4. Performances of different schemes versus coordinated circuit power $p^{\text {cirCo }}$ (non-selection). The network is set as $M=4, K_{0}=16, K_{j}=4$ for all $j \in \mathcal{M}, N=8, L_{i}=4$ for all $i \in \mathcal{N}$. Users 1 and 2 are only served by the macro BS and $\mathcal{N}_{1}=\{3,4\}, \mathcal{N}_{2}=\{5\}, \mathcal{N}_{3}=\{6,7\}, \mathcal{N}_{4}=\{8\}, \overline{\mathbf{r}}=$ $[14.43,28.85,15.87,24.53,30.3,17.31,21.64,18.76]$ Mbits/s.

to cause a significant impact on the sum rate, and thus the average sum rate is more or less the same. ${ }^{4}$ On the other hand, energy efficiency and sum rate performances of the SPmin scheme increase with $\bar{R}$. This is due to the fact that data rate increases linearly with power in the low power regime. That is to say, a small increase of transmit power maybe suffice to satisfy small $\bar{R}$.

In the last experiment we explore the performance gains provided by joint designs of precoding and BS selection. Particularly, Fig. 6 illustrates the average energy efficiency obtained by EEmax, JPBS with BnB, and JPBS with lowcomplexity design schemes versus the number of small-cell BSs. The setup network described in the caption means that, as $M=3$, each user of $\{2,3,4\}$ lies in the coverage area of one small cell, i.e. $\mathcal{M}_{2}=\{1\}, \mathcal{M}_{3}=\{2\}, \mathcal{M}_{4}=\{3\}$; and as $M=4$ then $\mathcal{M}_{2}=\{1,4\}, \mathcal{M}_{3}=\{2\}, \mathcal{M}_{4}=\{3\}$; and so on. The error tolerance parameter of $\mathrm{BnB}$ is $\varepsilon_{\mathrm{B}}=0.0014$ (Mbits $\left./ \mathrm{mJ}\right)$. As can be observed in the figure, the energy efficiency of EEmax (i.e.,

\footnotetext{
${ }^{4}$ If $\bar{R}$ is large enough, the sum rate of SRmax may reduce because more transmit power is required to satisfy QoS of users who are under deep fading channels. Herein, parameter $\bar{R}$ is kept at small value to guarantee that the resulting problems are feasible.
}

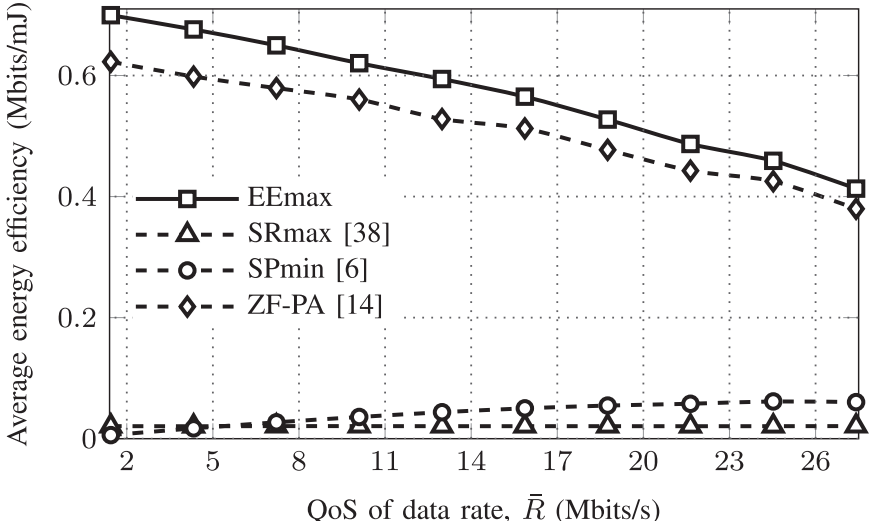

(a) Average energy-efficient performances

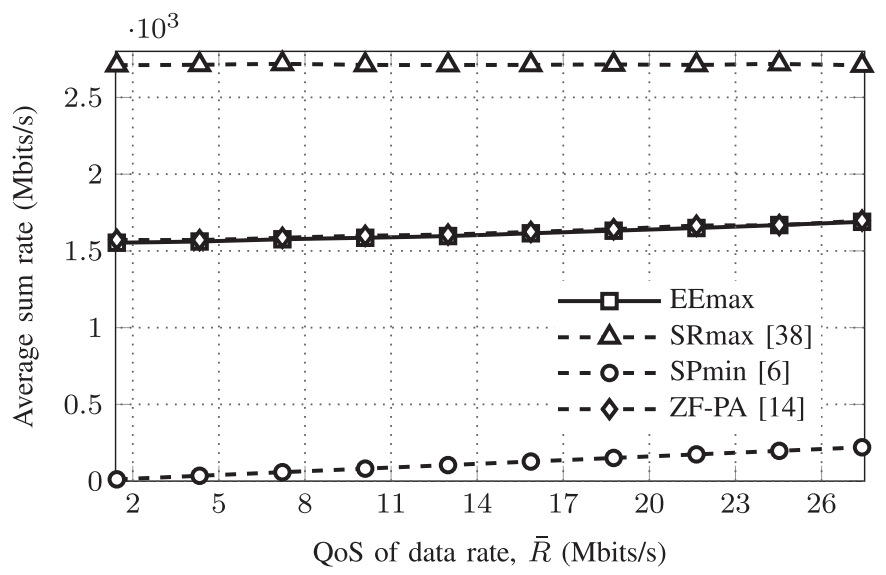

(b) Average sum rate performances

Fig. 5. Performances of different schemes versus QoS constraints of data rate (non-selection). The setting is the same as that of Fig. $4, \bar{R}_{i}=\bar{R}$ for all $i$, and $p^{\text {cirCo }}=7 \mathrm{dBm}$.

without BS selection) increases as users are covered by one small cell $(0<M \leq 3)$ and decreases as users are served by two small-cell BSs $(M \geq 4)$. This observation can be explained as follows. When a user is served only by the macro BS, due to the large distance between the macro BS and the user, macro BS needs an enormous amount of transmit power to satisfy the user's QoS constraint. As more small-cell BSs are available, the distance between a small-cell BS and a user becomes small, and thus small-cell BSs can increase the data rate with small transmit power and can help the macro BS reduces its transmit power. We note that an increase in the number of small-cell BSs provides more spatial diversity gains, but the gains may not compensate for additional circuit powers (i.e. $p_{j}^{\text {cirTx }}$ and $\left.p^{\text {cirCo }}\right)$. This is actually the case when $M \geq 4$ and BS selection becomes the key to improving energy efficiency in such cases. As can be seen, JPBS improves the network energy efficiency significantly, especially when the number of small-cell BSs is large. We also notice that the network energy efficiency of the proposed JPBS designs start reducing after a certain number of small-cell BSs. This is due to the fact that it requires a great amount of extra circuit power (i.e. $p^{\mathrm{cirSw}}$ ) to turn off the unselected BSs. Fig. 6 also indicates that the proposed low-complexity design for the JPBS problem is a near-optimal solution, making it appealing to practical applications. 


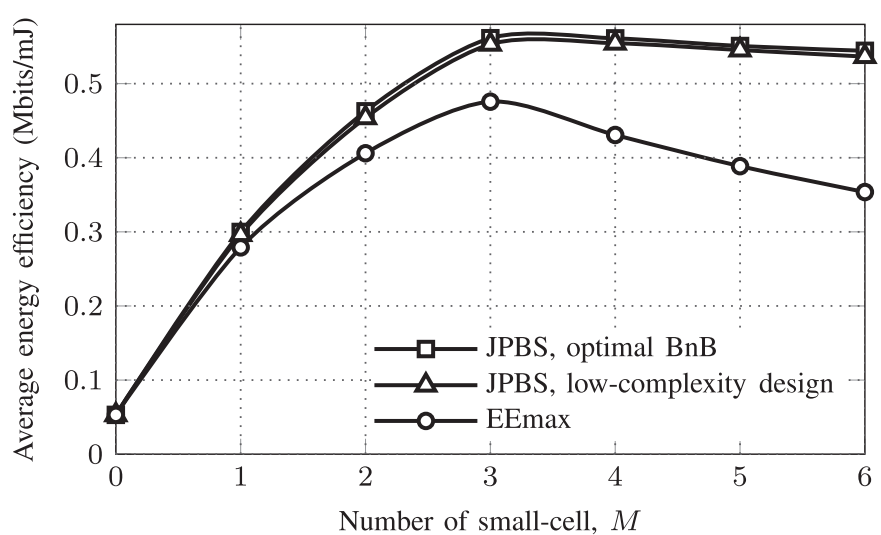

Fig. 6. Average energy efficiency of EEmax, JPBS with BnB, and JPBS with low-complexity design schemes versus the number of small-cell BSs. Other simulation parameters are as follows: $K_{0}=10, K_{j}=4, N=4, L_{i}=2$; user 1 is only served by the macro BS; $\mathcal{M}_{2}=\{1,4\}, \mathcal{M}_{3}=\{2,5\}, \mathcal{M}_{4}=\{3,6\}$, $\overline{\mathbf{r}}=[14.43,28.85,15.87,24.53] \mathrm{Mbits} / \mathrm{s}$, and $p^{\mathrm{cirCo}}=20 \mathrm{dBm}$.

\section{CONCLUSION}

We have addressed the energy-efficient precoder design for small-cell networks where nodes are equipped multiple antennas. In particular, we have formulated the EEmax problem as a fractional program with sum power and per-antenna power constraints and user-specific QoS constraints. Then, the problem has been solved by a centralized method based on Dinkelbach's algorithm. After that the EEmax problem has been transformed to a convex equivalent problem which is suitable for decentralized algorithms. In this regard we have proposed a distributed algorithm to solve the equivalent problem based on the ADMM. Furthermore, to enhance the energy efficiency of the network, we have investigated the problem of joint precoder design and BS selection, which is a mixed integer nonlinear program. An optimal solution, which is developed by customizing branch-and-bound method, has been presented. For real time applications we have also developed a low-complexity design which yields a near-optimal performance in polynomial time. The proposed algorithms has been evaluated by numerical experiments.

\section{APPENDIX}

We begin the proof of Lemma 1 by noting that the constraint $\left[\begin{array}{cc}\mathbf{I}+\tilde{\mathbf{H}}_{i} \boldsymbol{\Psi}_{i} \tilde{\mathbf{H}}_{i}^{H} & \boldsymbol{\Lambda}_{i} \\ \boldsymbol{\Lambda}_{i}^{H} & \operatorname{diag}\left(\mathbf{q}_{i}\right)\end{array}\right] \succeq \mathbf{0}$ is satisfied amounts to $\mid \mathbf{I}+$ $\tilde{\mathbf{H}}_{i} \boldsymbol{\Psi}_{i} \tilde{\mathbf{H}}_{i}^{H} \mid \geq \prod_{l=1}^{L_{i}} q_{i l}$ where $\mathbf{q}_{i}=\operatorname{diag}\left(\boldsymbol{\Lambda}_{i}\right)$ [31, Section 3.2]. Then, combining with the constraint $\left(\prod_{l=1}^{L_{i}} q_{i l}\right)^{1 / L_{i}} \geq e^{\bar{R}_{i} / L_{i}}$, we have (7b). Furthermore, in the light of [31, Section 3.2], we also have the fact that, for a given positive definite matrix $\mathbf{I}+\tilde{\mathbf{H}}_{i} \boldsymbol{\Psi}_{i} \tilde{\mathbf{H}}_{i}^{H}$, there always exists a lower triangle matrix $\boldsymbol{\Lambda}_{i}$ that satisfies the constraints $\left[\begin{array}{cc}\mathbf{I}+\tilde{\mathbf{H}}_{i} \boldsymbol{\Psi}_{i} \tilde{\mathbf{H}}_{i}^{H} & \boldsymbol{\Lambda}_{i} \\ \boldsymbol{\Lambda}_{i}^{H} & \operatorname{diag}\left(\mathbf{q}_{i}\right)\end{array}\right] \succeq \mathbf{0}$ and $\left|\mathbf{I}+\tilde{\mathbf{H}}_{i} \boldsymbol{\Psi}_{i} \tilde{\mathbf{H}}_{i}^{H}\right|=\prod_{l=1}^{L_{i}} q_{i l}$. Now, we prove that the inequality $\left|\mathbf{I}+\tilde{\mathbf{H}}_{i} \boldsymbol{\Psi}_{i} \tilde{\mathbf{H}}_{i}^{H}\right| \geq \prod_{l=1}^{L_{i}} q_{i l}$ is active at optimum by contradiction. Suppose that $\left|\mathbf{I}+\tilde{\mathbf{H}}_{i} \boldsymbol{\Psi}_{i} \tilde{\mathbf{H}}_{i}^{H}\right|>\prod_{l=1}^{L_{i}} q_{i l}$ at optimum. However we can appropriately choose $\boldsymbol{\Lambda}_{i}^{\prime}$ such that $\mid \mathbf{I}+$
$\tilde{\mathbf{H}}_{i} \boldsymbol{\Psi}_{i} \tilde{\mathbf{H}}_{i}^{H} \mid=\prod_{l=1}^{L_{i}} q_{i l}^{\prime}$, and thus $\prod_{l=1}^{L_{i}} q_{i l}^{\prime}>\prod_{l=1}^{L_{i}} q_{i l}$, which means $\sum_{l=1}^{L_{i}} \log q_{i l}^{\prime}-\tau P^{\text {Total }}>\sum_{l=1}^{L_{i}} \log q_{i l}-\tau P^{\text {Total }}$. This contradicts with the fact that $\boldsymbol{\Lambda}_{i}$ is optimal, and thus completes the proof.

\section{REFERENCES}

[1] Small Cell Forum, "W-CDMA open access small cells: Architecture, requirements and dependencies," White Paper SCF038, Feb. 2012 [Online]. Available: http://scf.io/en/index.php

[2] J. Hoydis, M. Kobayashi, and M. Debbah, "Green small-cell networks," IEEE Veh. Technol. Mag., vol. 6, no. 1, pp. 37-43, Mar. 2011.

[3] Y. S. Soh, T. Quek, M. Kountouris, and H. Shin, "Energy efficient heterogeneous cellular networks," IEEE J. Sel. Areas Commun., vol. 31, no. 5, pp. 840-850, May 2013.

[4] M. Hong, R. Sun, H. Baligh, and Z.-Q. Luo, "Joint base station clustering and beamformer design for partial coordinated transmission in heterogeneous networks," IEEE J. Sel. Areas Commun., vol. 31, no. 2, pp. 226-240, Feb. 2013.

[5] E. Bjornson, M. Kountouris, and M. Debbah, "Massive MIMO and small cells: Improving energy efficiency by optimal soft-cell coordination," in Proc. 20th Int. Conf. Telecommun. (ICT), May 2013, pp. 1-5.

[6] Z. Xu, C. Yang, G. Li, Y. Liu, and S. Xu, "Energy-efficient CoMP precoding in heterogeneous networks," IEEE Trans. Signal Process., vol. 62, no. 4, pp. 1005-1017, Feb. 2014.

[7] D. Nguyen, L. Tran, P. Pirinen, and M. Latva-aho, "On the spectral efficiency of full-duplex small cell wireless systems," IEEE Trans. Wireless Commun., vol. 13, no. 9, pp. 4896-4910, Sep. 2014.

[8] G. Miao, N. Himayat, and G. Li, "Energy-efficient link adaptation in frequency-selective channels," IEEE Trans. Commun., vol. 58, no. 2, pp. 545-554, Feb. 2010.

[9] D. Nguyen, L.-N. Tran, P. Pirinen, and M. Latva-aho, "Precoding for full duplex multiuser MIMO systems: Spectral and energy efficiency maximization," IEEE Trans. Signal Process., vol. 61, no. 16, pp. 4038-4050, Aug. 2013.

[10] E. Bjornson, L. Sanguinetti, J. Hoydis, and M. Debbah, "Optimal design of energy-efficient multi-user MIMO systems: Is massive MIMO the answer?" IEEE Trans. Wireless Commun., vol. 14, no. 6, pp. 3059-3075, Jun. 2015.

[11] B. Bejar Haro, S. Zazo, and D. Palomar, "Energy efficient collaborative beamforming in wireless sensor networks," IEEE Trans. Signal Process., vol. 62, no. 2, pp. 496-510, Jan. 2014.

[12] S. He, Y. Huang, S. Jin, and L. Yang, "Coordinated beamforming for energy efficient transmission in multicell multiuser systems," IEEE Trans. Commun., vol. 61, no. 12, pp. 4961-4971, Dec. 2013.

[13] S. He, Y. Huang, L. Yang, and B. Ottersten, "Coordinated multicell multiuser precoding for maximizing weighted sum energy efficiency," IEEE Trans. Signal Process., vol. 62, no. 3, pp. 741-751, Feb. 2014.

[14] X. Wang, P. Zhu, B. Sheng, and X. You, "Energy-efficient downlink transmission in multi-cell coordinated beamforming systems," in Proc. IEEE Wireless Commun. Netw. Conf. (WCNC), Apr. 2013, pp. 2554-2558.

[15] C. Isheden, Z. Chong, E. Jorswieck, and G. Fettweis, "Framework for link-level energy efficiency optimization with informed transmitter," IEEE Trans. Wireless Commun., vol. 11, no. 8, pp. 2946-2957, Aug. 2012.

[16] H. Weingarten, Y. Steinberg, and S. Shamai, "The capacity region of the Gaussian multiple-input multiple-output broadcast channel," IEEE Trans. Inf. Theory, vol. 52, no. 9, pp. 3936-3964, Sep. 2006.

[17] Q. Spencer, A. Swindlehurst, and M. Haardt, "Zero-forcing methods for downlink spatial multiplexing in multiuser MIMO channels," IEEE Trans. Signal Process., vol. 52, no. 2, pp. 461-471, Feb. 2004.

[18] S. Christensen, R. Agarwal, E. Carvalho, and J. Cioffi, "Weighted sumrate maximization using weighted MMSE for MIMO-BC beamforming design," IEEE Trans. Wireless Commun., vol. 7, no. 12, pp. 4792-4799, Dec. 2008.

[19] A. Charnes and W. W. Cooper, "Programming with linear fractional functionals," Nav. Res. Logist. Quart., vol. 9, nos. 3-4, pp. 181-186, 1962.

[20] S. Boyd, N. Parikh, E. Chu, B. Peleato, and J. Eckstein, "Distributed optimization and statistical learning via the alternating direction method of multipliers," Found. Trends Mach. Learn., vol. 3, no. 1, pp. 1-122, 2011.

[21] J. Lee and S. Leyffer, Mixed Integer Nonlinear Programming. New York, NY, USA: Springer, 2011. 
[22] D. P. Bertsekas, Nonlinear Programming, 3rd ed. Belmont, MA, USA: Athena Scientific, 2008.

[23] L.-N. Tran, M. Juntti, M. Bengtsson, and B. Ottersten, "Beamformer designs for MISO broadcast channels with zero-forcing dirty paper coding," IEEE Trans. Wireless Commun., vol. 12, no. 3, pp. 1173-1185, Mar. 2013.

[24] L.-N. Tran, M. Bengtsson, and B. Ottersten, "Iterative precoder design and user scheduling for block-diagonalized systems," IEEE Trans. Signal Process., vol. 60, no. 7, pp. 3726-3739, Jul. 2012.

[25] L.-N. Tran, M. Juntti, M. Bengtsson, and B. Ottersten, "Weighted sum rate maximization for MIMO broadcast channels using dirty paper coding and zero-forcing methods," IEEE Trans. Commun., vol. 61, no. 6, pp. 2362-2373, Jun. 2013.

[26] S. Boyd and L. Vandenberghe, Convex Optimization, 1st ed. Cambridge, U.K.: Cambridge Univ. Press, 2004.

[27] W. Dinkelbach, "On nonlinear fractional programming," Manage. Sci., vol. 13, no. 7, pp. 492-498, Oct. 1967.

[28] Q. Spencer, A. Swindlehurst, and M. Haardt, "Zero-forcing methods for downlink spatial multiplexing in multiuser MIMO channels," IEEE Trans. Signal Process., vol. 52, no. 2, pp. 461-471, Feb. 2004.

[29] D. P. Bertsekas, A. Nedic, and A. E. Ozdagla, Convex Analysis and Optimization, Belmont, MA, USA: Athena Scientific, 2003.

[30] L. Vandenberghe, S. Boyd, and S.-P. Wu, "Determinant maximization with linear matrix inequality constraints," SIAM J. Matrix Anal. Appl., vol. 19 , no. 2, pp. 499-533, 1998.

[31] A. Ben-Tal and A. Nemirovski, Lectures on Modern Convex Optimization. Philadelphia, PA, USA: SIAM, 2001.

[32] K. C. Toh, M. Todd, and R. H. Tütüncü, "SDPT3-A MATLAB software package for semidefinite programming," Optim. Method. Softw., vol. 11, pp. 545-581, 1999.

[33] D. Palomar and M. Chiang, "A tutorial on decomposition methods for network utility maximization," IEEE J. Sel. Areas Commun., vol. 24, no. 8, pp. 1439-1451, Aug. 2006.

[34] Q.-D. Vu, L.-N. Tran, M. Juntti, and E.-K. Hong, "Energy-efficient bandwidth and power allocation for multi-homing networks," IEEE Trans. Signal Process., vol. 63, no. 7, pp. 1684-1699, Apr. 2015.

[35] L. Xiao and S. Boyd, "Fast linear iterations for distributed averaging," Syst. Control Lett., vol. 53, pp. 65-78, 2004.

[36] M. S. Lobo, L. Vandenberghe, S. Boyd, and H. Lebret, "Application of second-order cone programming," Linear Algebra Appl., vol. 284, pp. 193-228, Nov. 1998.

[37] Y. Cheng, M. Pesavento, and A. Philipp, "Joint network optimization and downlink beamforming for CoMP transmissions using mixed integer conic programming," IEEE Trans. Signal Process., vol. 61, no. 16, pp. 3972-3987, Aug. 2013.

[38] R. Zhang, "Cooperative multi-cell block diagonalization with per-basestation power constraints," IEEE J. Sel. Areas Commun., vol. 28, no. 9, pp. 1435-1445, Dec. 2010.

[39] S. Cui, A. Goldsmith, and A. Bahai, "Energy-constrained modulation optimization," IEEE Trans. Wireless Commun., vol. 4, no. 5, pp. 23492360, Sep. 2005.

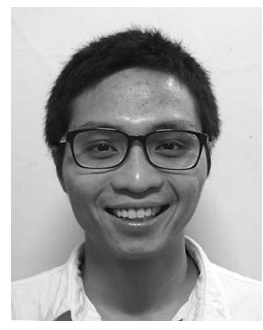

Quang-Doanh Vu received the B.S. degree in electrical engineering from Ho Chi Minh National University of Technology, Ho Chi Minh City, Vietnam, and the M.S. and Ph.D. degrees in radio engineering from Kyung Hee University, Seoul, South Korea, in 2010, 2012, and 2015, respectively. $\mathrm{He}$ is currently a Postdoctoral Researcher with the Centre for Wireless Communications, Department of Communications Engineering, University of Oulu, Oulu, Finland. His research interests include resource allocation, energy-efficient communications, multiuser MIMO systems, and wireless power transfer.

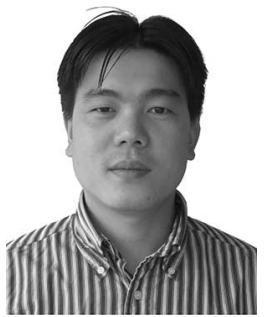

Le-Nam Tran (M'10) received the B.S. degree in electrical engineering from Ho Chi Minh City University of Technology, Ho Chi Minh City, Vietnam, and the M.S. and Ph.D. degrees in radio engineering from Kyung Hee University, Seoul, South Korea, in 2003, 2006, and 2009, respectively. In 2009, he joined the Department of Electrical Engineering, Kyung Hee University, as a Lecturer. From 2010 to 2011, he was a Postdoctoral Fellow with the Signal Processing Laboratory, ACCESS Linnaeus Centre, KTH Royal Institute of Technology, Stockholm, Sweden. From 2011 to 2014, he was with the Centre for Wireless Communications, Department of Communications Engineering, University of Oulu, Oulu, Finland. He is currently a Lecturer with the Department of Electronic Engineering, Maynooth University, Kildare, Ireland. His research interests include multiuser MIMO systems, energy-efficient communications, full-duplex transmission, and applications of optimization techniques.

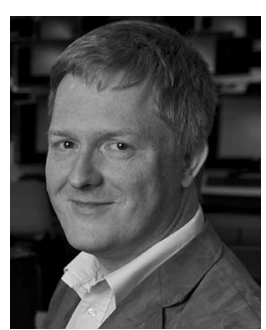

Ronan Farrell (M'93) received the B.E. and Ph.D. degrees in electronic engineering from the University College Dublin, Dublin, Ireland, in 1993 and 1998, respectively. He is currently a Senior Lecturer with the National University of Ireland, Dublin, Ireland. His research interests include physical layer communication technologies, in particular signal processing for adaptive receivers, power amplifiers and active antenna arrays. He is currently a Senior Investigator, responsible for radio technologies in the SFI-funded Centre for Telecommunications Research and the SFI CONNECT Centre for the Internet of Things.

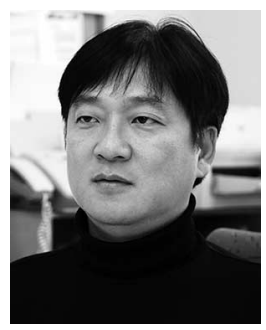

Een-Kee Hong (M'99-SM'09) received the B.S., M.S., and Ph.D. degrees in electrical engineering from Yonsei University, Seoul, South Korea, in 1989, 1991, and 1995, respectively. He was a Senior Research Engineer with SK Telecom from September 1995 to February 1999 and a Visiting Senior Engineer at NTT DoCoMo from October 1997 to December 1998. From 2006 to 2007, he was a Visiting Professor at Oregon State University, Corvallis, OR, USA. He has been a Professor since 1999, and served as the Vice Dean with College of Electronics and Information Engineering, Kyung Hee University, Seoul, South Korea. His research interests include physical layer in wireless communication, spectrum engineering, and cross-layer optimization. He was the recipient of the Best Paper Award, Institute of Information Technology Assessment, and Haedong Best Paper Award, KICS. 